Check for

updates

Cite as

Nano-Micro Lett.

(2021) 13:202

Received: 26 July 2021

Accepted: 31 August 2021

Published online: 26 September 2021

(C) The Author(s) 2021

\section{MOF-Derived $\mathrm{ZnS}$ Nanodots $/ \mathrm{Ti}_{3} \mathrm{C}_{2} \mathrm{~T}_{x}$ MXene Hybrids Boosting Superior Lithium Storage Performance}

\author{
Bin $\mathrm{Cao}^{1}$, Huan $\mathrm{Liu}^{1,2}{ }^{凶}$, Xin Zhang ${ }^{1}$, Peng Zhang ${ }^{1}$, Qizhen Zhu ${ }^{1}$, Huiling $\mathrm{Du}^{2}$,
} Lianli Wang ${ }^{2}$, Rupeng Zhang ${ }^{1}$, Bin Xu ${ }^{1} \bowtie$

\title{
HIGHLIGHTS
}

- The unique 0D-2D ZnS nanodots/Ti ${ }_{3} \mathrm{C}_{2} \mathrm{~T}_{x}$ MXene hybrids with strong interfacial interaction enable to achieve stable cyclability and excellent rate performance for lithium storage.

- The lithium storage mechanism of $\mathrm{ZnS}$ is clarified and new insights into phase transition mechanism are proposed.

- The strong interfacial interaction between ZnS nanodots and MXene nanosheets at the ZnS-MXene heterointerface exhibits high lithium adsorption capability, enhanced interfacial electron transfer, and low lithium diffusion energy barrier.

ABSTRACT ZnS has great potentials as an anode for lithium storage because of its high theoretical capacity and resource abundance; however, the large volume expansion accompanied with structural collapse and low conductivity of $\mathrm{ZnS}$ cause severe capacity fading and inferior rate capability during lithium storage. Herein, 0D-2D $\mathrm{ZnS}$ nanodots $/ \mathrm{Ti}_{3} \mathrm{C}_{2} \mathrm{~T}_{x}$ MXene hybrids are prepared by anchoring $\mathrm{ZnS}$ nanodots on $\mathrm{Ti}_{3} \mathrm{C}_{2} \mathrm{~T}_{x}$ MXene nanosheets through coordination modulation between MXene and MOF precursor (ZIF-8) followed with sulfidation. The MXene substrate

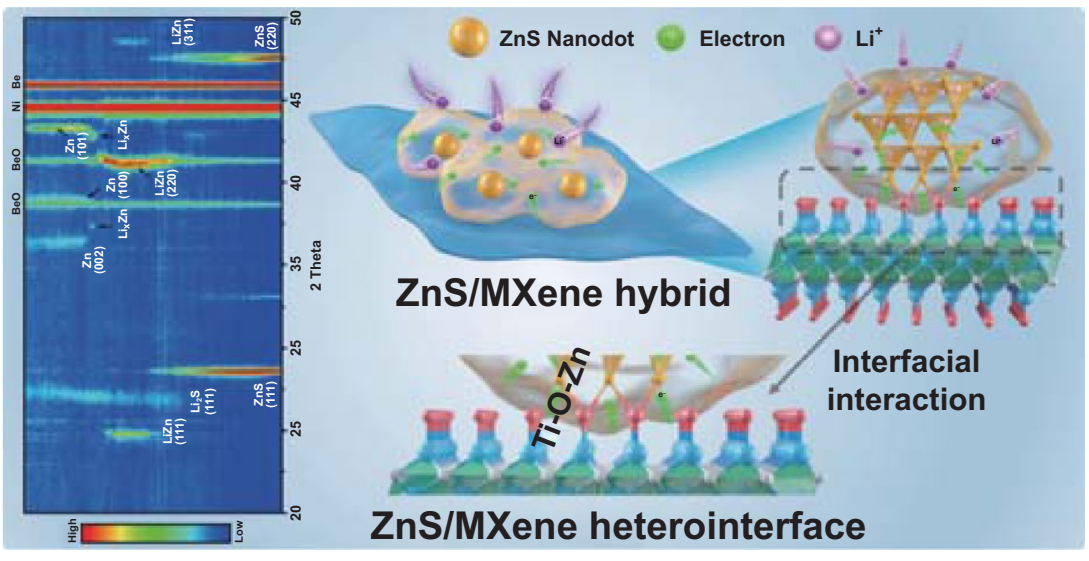
coupled with the $\mathrm{ZnS}$ nanodots can synergistically accommodate volume variation of $\mathrm{ZnS}$ over charge-discharge to realize stable cyclability. As revealed by XPS characterizations and DFT calculations, the strong interfacial interaction between ZnS nanodots and MXene nanosheets can boost fast electron/lithium-ion transfer to achieve excellent electrochemical activity and kinetics for lithium storage. Thereby, the as-prepared $\mathrm{ZnS}$ nanodots/MXene hybrid exhibits a high capacity of $726.8 \mathrm{mAh} \mathrm{g}^{-1}$ at $30 \mathrm{~mA} \mathrm{~g}^{-1}$, superior cyclic stability (462.8 mAh g-1 after 1000 cycles at $0.5 \mathrm{~A} \mathrm{~g}^{-1}$ ), and excellent rate performance. The present results provide new insights into the understanding of the lithium storage mechanism of $\mathrm{ZnS}$ and the revealing of the effects of interfacial interaction on lithium storage performance enhancement.

KEYWORDS $\mathrm{Ti}_{3} \mathrm{C}_{2} \mathrm{~T}_{x}$ MXene; MOF; Interfacial interaction; Heterointerface; ZnS; Lithium-ion batteries

Huan Liu, huanliu@xust.edu.cn; Bin Xu, xubin@mail.buct.edu.cn

1 State Key Laboratory of Organic-Inorganic Composites, Beijing Key Laboratory of Electrochemical Process and Technology for Materials, Beijing

University of Chemical Technology, Beijing 100029, People's Republic of China

2 College of Materials Science and Engineering, Xi' an University of Science and Technology, Xi' an 710054, People's Republic of China 


\section{Introduction}

Electrochemical energy storage is a rapidly growing research field due to the ever-increasing requirements for smart grids and electric/hybrid vehicles. Lithium-ion batteries (LIBs) are currently the dominant energy storage system due to their high energy and power density, long cycle life, and low self-discharge rate $[1,2]$. Nevertheless, the present LIBs technology faces challenges to achieve a higher energy density and power output because the currently used graphite anodes have a low theoretical capacity $\left(372 \mathrm{mAh} \mathrm{g}^{-1}\right)$ and unsatisfied rate performance. In addition, the low lithiation potential $\left(\sim 0.1 \mathrm{~V} v s . \mathrm{Li}^{+} / \mathrm{Li}\right)$ of graphite enables the risk of lithium dendrites formation, which may trigger internal short and thus lead to safety concern [3,4]. Transition metal sulfides (TMSs) [5-7], such as $\mathrm{MoS}_{2}$ [8, 9], $\mathrm{SnS}_{2}[10,11]$, $\mathrm{CoS}$ [12], and $\mathrm{ZnS}$ [13], have received considerable attention as promising candidate anode materials to replace graphite on account of their high specific capacity and moderate working potential. Among TMSs, $\mathrm{ZnS}$ is one of the most attractive anode candidates for lithium storage because of its high theoretical lithium storage capacity, non-toxicity, and low cost. Based on the conversion and alloying reactions from $\mathrm{ZnS}$ to $\mathrm{LiZn}$ alloy and $\mathrm{Li}_{2} \mathrm{~S}$, a high theoretical specific capacity of $825 \mathrm{mAh} \mathrm{g}^{-1}$ can be achieved $[14,15]$. Nevertheless, bulk ZnS suffers drastic capacity fading, which is caused by its substantial volume change during lithium storage and the consequent pulverization of $\mathrm{ZnS}$ particles. In addition, the low conductivity of $\mathrm{ZnS}$ leads to sluggish kinetics for lithium/electron transfer, which limits the rate performance for lithium storage. It is worth noting that the $\mathrm{ZnS}$ materials lacking conductive matrix and electron transfer pathway also usually suffers from unsatisfactory electrochemical activities $[16,17]$.

Recently, significant efforts have been devoted to improve the electrochemical performance of $\mathrm{ZnS}$ by using nanosized $\mathrm{ZnS}$ or designing nanostructures, such as $\mathrm{ZnS}$ quantum dots [13], ZnS nanotubes [18], and ZnS nanocrystals [19], which is an effective way to reduce the absolute volume expansion and shorten the diffusion pathway in solid phase during lithiation/delithiation. Anchoring $\mathrm{ZnS}$ on conductive matrix is another effective way to improve the electrochemical performance of $\mathrm{ZnS}$ as it can not only improve the conductivity but also accommodate the volume change. Several conductive matrixes including graphene $[5,6,16]$, porous carbon $[17$,
20, 21], and carbon nanotubes [22] were adopted to combine with $\mathrm{ZnS}$ for improving electrochemical performance. MXenes, a new family of the 2D materials, have already been proved to be promising candidate as electrode materials for electrochemical energy storage devices [23-31], such as LIBs and supercapacitors. Among the large family of MXenes, $\mathrm{Ti}_{3} \mathrm{C}_{2} \mathrm{~T}_{x}$ [32] is the most widely studied one for electrochemical energy storage owing to its high electronic conductivity (up to $2.4 \times 10^{4} \mathrm{~S} \mathrm{~cm}^{-1}$ ) [33], hydrophilic surfaces and high density [34]. The theoretical conductivity of the $\mathrm{Ti}_{3} \mathrm{C}_{2} \mathrm{~T}_{x}$ MXene is even higher than carbon nanotubes and graphene because of its metal carbide core layers [35]. Hence, using MXene as an excellent conductive matrix is very effective to improve the electrochemical performance of active materials, such as TMS and other active materials (such as $\mathrm{SnO}_{2}$ [36, 37], $\mathrm{Co}_{3} \mathrm{O}_{4}$ [38], P [27, 39, 40], and $\mathrm{Si}$ $[41,42])$ with low intrinsic conductivity. Inspired by the above discussion, $\mathrm{Ti}_{3} \mathrm{C}_{2} \mathrm{~T}_{x}$ MXene is considered as a superior conducting substrate to improve the electrochemical performances of $\mathrm{ZnS}$.

In this work, the 0D-2D ZnS nanodots $/ \mathrm{Ti}_{3} \mathrm{C}_{2} \mathrm{~T}_{x}$ MXene nanosheets hybrids (ZnSMX) with excellent lithium storage performance were prepared by coordination modulation between MXene and ZIF-8 precursor followed with sulfidation. In $\mathrm{ZnSMX}$ hybrids, the nanodot morphology of $\mathrm{ZnS}$ is beneficial to reduce the lithium diffusion distance in solid phase and enhance the electrochemical kinetics. Furthermore, the $\mathrm{Ti}_{3} \mathrm{C}_{2} \mathrm{~T}_{x}$ MXene nanosheets serving as $2 \mathrm{D}$ substrate not only provide fast electron transfer pathways, but also prevent the aggregation of $\mathrm{ZnS}$ nanodots and accommodate the volume change of $\mathrm{ZnS}$ during charge-discharge. More importantly, ZnS nanodots can be in situ locked tightly on the surface of 2D MXene nanosheets through interfacial Ti-O-Zn bond, thereby effectively suppressing the detachment of $\mathrm{ZnS}$ nanodots from the conducting MXene substrate. According to XPS characterizations and DFT calculation results, the charge density trends to accumulate at $\mathrm{ZnS}$ side in ZnS-MXene heterointerface, which indicates strong interfacial interaction and may enhance the electron mobility and electronic conductivity of $\mathrm{ZnS}$. Moreover, $\mathrm{ZnS}-\mathrm{MXene}$ heterointerface exhibits strong lithium adsorption capability and low diffusion energy barrier, which favors for achieving excellent electrochemical performances. Benefiting from these advantages, the 0D-2D ZnS/MXene hybrid shows a high reversible capacity of $726.8 \mathrm{mAh} \mathrm{g}^{-1}$ at $30 \mathrm{~mA} \mathrm{~g}^{-1}$, 
and long-term cycling stability of $462.8 \mathrm{mAh} \mathrm{g}^{-1}$ after 1000 cycles at $0.5 \mathrm{~A} \mathrm{~g}^{-1}$ without obvious capacity fading. The results imply that optimizing the interfacial interaction between $\mathrm{ZnS}$ nanodots and $\mathrm{Ti}_{3} \mathrm{C}_{2} \mathrm{~T}_{x}$ nanosheets plays an important role for boosting lithium storage performances.

\section{Experimental Section}

\subsection{Synthesis of $\mathrm{Ti}_{3} \mathrm{C}_{2} \mathrm{~T}_{x}$ MXene Nanosheets}

$1.0 \mathrm{~g}$ of $\mathrm{Ti}_{3} \mathrm{AlC}_{2}$ (11Technology Co., Ltd) was slowly added to a mixed solution of $0.99 \mathrm{~g} \mathrm{LiF}$ (Alfa Aesar, 98.5\%) and $10 \mathrm{~mL} \mathrm{HCl}(12 \mathrm{M})$, and the mixture was stirred at $35^{\circ} \mathrm{C}$ for $24 \mathrm{~h}$. Afterward, the multilayered $\mathrm{Ti}_{3} \mathrm{C}_{2} \mathrm{~T}_{x}$ was carefully washed and centrifuged with deionized water for 5 times at a speed of $5000 \mathrm{rpm}$ until the $\mathrm{pH}$ value of the supernatant reached $\sim 6$. The resulting $\mathrm{Ti}_{3} \mathrm{C}_{2} \mathrm{~T}_{x}$ suspension was mixed with $200 \mathrm{~mL}$ deionized water and ultrasonicated for $1 \mathrm{~h}$ under argon atmosphere. Followed by centrifugation at $3500 \mathrm{rpm}$ for $1 \mathrm{~h}$, the aqueous suspension containing MXene nanosheets was obtained. The water in aqueous MXene dispersion was replaced with methanol by solvent exchange method. Finally, $\mathrm{Ti}_{3} \mathrm{C}_{2} \mathrm{~T}_{x}$ MXene methanol dispersion was obtained for later use.

\subsection{Synthesis of ZIF-8/MXene}

Zinc nitrate hexahydrate methanol solution $(0.9 \mathrm{mmol}$ $\mathrm{Zn}\left(\mathrm{NO}_{3}\right)_{2} \cdot 6 \mathrm{H}_{2} \mathrm{O}$, in $20 \mathrm{~mL}$ methanol) was added into the $\mathrm{Ti}_{3} \mathrm{C}_{2} \mathrm{~T}_{x}$ methanol dispersion $\left(25 \mathrm{~mL}, 2 \mathrm{mg} \mathrm{mL}^{-1}\right)$ under stirring for $30 \mathrm{~min}$. Then, 2-methylimidazole (2-MeIM) was dissolved in $20 \mathrm{~mL}$ methanol. Subsequently, the 2-MeIM methanol solution was poured into the as-prepared zinc nitrate hexahydrate/ $\mathrm{Ti}_{3} \mathrm{C}_{2} \mathrm{~T}_{x}$ MXene methanol dispersion ( $\mathrm{Zn}: 2-\mathrm{MeIM}=1: 16 \mathrm{M}$ ) under magnetic stirring for $1 \mathrm{~h}$ at room temperature. After filtrating the suspension on Celgard 3501 membrane and further washing with methanol three times, the sample labeled as ZIF-8/MXene-0.9 was obtained and then dried at $80{ }^{\circ} \mathrm{C}$ in a vacuum oven overnight. The ZIF-8/MXene-2 precursor with a higher ZIF-8 content was prepared by using $2 \mathrm{mmol} \mathrm{Zn}\left(\mathrm{NO}_{3}\right)_{2} \cdot 6 \mathrm{H}_{2} \mathrm{O}$ under similar preparation conditions.

\subsection{Synthesis of ZnS/MXene Hybrids}

The ZIF-8/MXene- $(0.9,2)$ precursor and thioacetamide (TAA, $10 \mathrm{mmol}$ ) were added to a methanol/water mixed solution (volume ratio of $100 \mathrm{~mL} / 10 \mathrm{~mL}$ ), and stirred under an argon atmosphere for $30 \mathrm{~min}$. Subsequently, the mixed solution was refluxed and stirred at $60^{\circ} \mathrm{C}$ for $6 \mathrm{~h}$. The sulfur substitution reaction was protected under nitrogen atmosphere until the reaction was finished. Then, the black solid resultant was collected by filtration, washed with deionized water and dried. The mass ratio of $\mathrm{ZnS}$ derived from the ZIF-8/MXene-0.9 and ZIF-8/MXene-2 was calculated as 64 and $80 \mathrm{wt} \%$, respectively. Thus, the corresponding $\mathrm{ZnS} /$ MXene hybrids were denoted as ZnSMX64 and ZnSMX80. For comparison, the pure $\mathrm{ZnS}$ was prepared with the same method just without adding MXene dispersion.

\subsection{Material Characterization}

The morphology and structure of the samples were characterized using scanning electron microscope (SEM, Hitachi S4800), transmission electron microscopy (TEM, Hitachi HT7700) and atomic force microscopy (AFM, Bruker edge). The XRD patterns of the samples were collected by using Bruker D8 Advanced XRD with $\mathrm{Cu} \mathrm{K} \alpha$ radiation source. Raman spectra were obtained using a Raman spectrometer (Renishaw inVia Reflex with $633 \mathrm{~nm}$ laser excitation). XPS measurements were taken on a Thermo Fisher ESCALAB $\mathrm{Xi}^{+}$. The Zeta potential was conducted using a Zetasizer Nano ZSE apparatus. The specific surface area and porosity parameters were determined by the nitrogen sorption/desorption using a Micromeritics ASAP2460 analyzer.

\subsection{Electrochemical Measurements}

The electrodes were prepared by mixing active material (80 wt\%), conductive carbon (Super P, $10 \mathrm{wt} \%$ ), and carboxymethylcellulose (CMC, $10 \mathrm{wt} \%$ ) with water into an electrode slurry and then the slurry was cast onto a $\mathrm{Cu}$ foil and dried in a vacuum oven at $120{ }^{\circ} \mathrm{C}$ for $8 \mathrm{~h}$. The 
mass loading of electrodes was controlled as $1 \mathrm{mg} \mathrm{cm}^{-2}$. Lithium foil and glass fiber membrane were used as the counter electrode and separator, respectively, and $1 \mathrm{M}$ $\mathrm{LiPF}_{6}$ in ethylene carbonate (EC)/diethylcarbonate (DEC) (1:1 in volume) was used as the electrolyte. 2025 coin-type cells were assembled in an argon-filled glove box (with $\mathrm{O}_{2}$ and $\mathrm{H}_{2} \mathrm{O}<0.1 \mathrm{ppm}$ ) to evaluate the electrochemical performance on Neware batteries testing systems (CT4008T-5V50mA-164, Shenzhen, China). The capacity was calculated based on the mass of the $\mathrm{ZnS} / \mathrm{MXene}$ hybrids. Cyclic voltammetry (CV) curves were collected at different scan rates between 0.01 and $2.5 \mathrm{~V}$ on a DH7000 electrochemistry workstation (Jiangsu Donghua Testing Technology Co., Ltd.). The electrochemical impedance spectroscopy (EIS) measurements were taken within a frequency range of $100 \mathrm{kHz}-0.01 \mathrm{~Hz}$ with an amplitude of $10 \mathrm{mV}$. The GITT measurements were taken at a current density of $100 \mathrm{~mA} \mathrm{~g}^{-1}$ for $15 \mathrm{~min}$ followed by relaxation of $2 \mathrm{~h}$. The in situ XRD measurement was taken on Bruker D8 $(\mathrm{Cu} \mathrm{K} \alpha$ radiation, $40 \mathrm{kV}$ and $40 \mathrm{~mA}$ ) with an in situ cell equipped with Be window to allow X-ray passage (designed by
Tianjin AIDA hengsheng Science and Technology Development Co., Ltd.) under a scan rate of $4^{\circ} \mathrm{min}^{-1}$. The scan range was $2 \theta=20-50^{\circ}$, and the corresponding galvanostatic discharge-charge cycling was conducted at a current density of $100 \mathrm{~mA} \mathrm{~g}^{-1}$.

\section{Results and Discussion}

The preparation of $\mathrm{ZnS} / \mathrm{MXene}$ hybrids is schematically illustrated in Fig. 1. Initially, $\mathrm{Ti}_{3} \mathrm{AlC}_{2}$ powder was etched by $\mathrm{LiF}$ and $\mathrm{HCl}$ followed with exfoliation to achieve $\mathrm{Ti}_{3} \mathrm{C}_{2} \mathrm{~T}_{x}$ MXene nanosheets with a lateral size of $500 \mathrm{~nm}$ (Fig. S1). The zeta potential of the prepared MXene sheets is $-36 \mathrm{mV}$ (Fig. S2), which is ascribed to the functional groups (e.g., $-\mathrm{F},-\mathrm{O},-\mathrm{OH})$ on the surface. The $\mathrm{Ti}_{3} \mathrm{C}_{2} \mathrm{~T}_{x}$ MXene was used as substrate for in situ nucleation and growth of the ZIF-8. After adding zinc nitrate hexahydrate methanol solution into MXene methanol dispersion, the positively charged $\mathrm{Zn}^{2+}$ was electrostatically absorbed on the surface of MXene nanosheets, and then, the $\mathrm{Zn}^{2+}$ acted as the central site to coordinate with the organic linker 2-MeIM, resulting in the

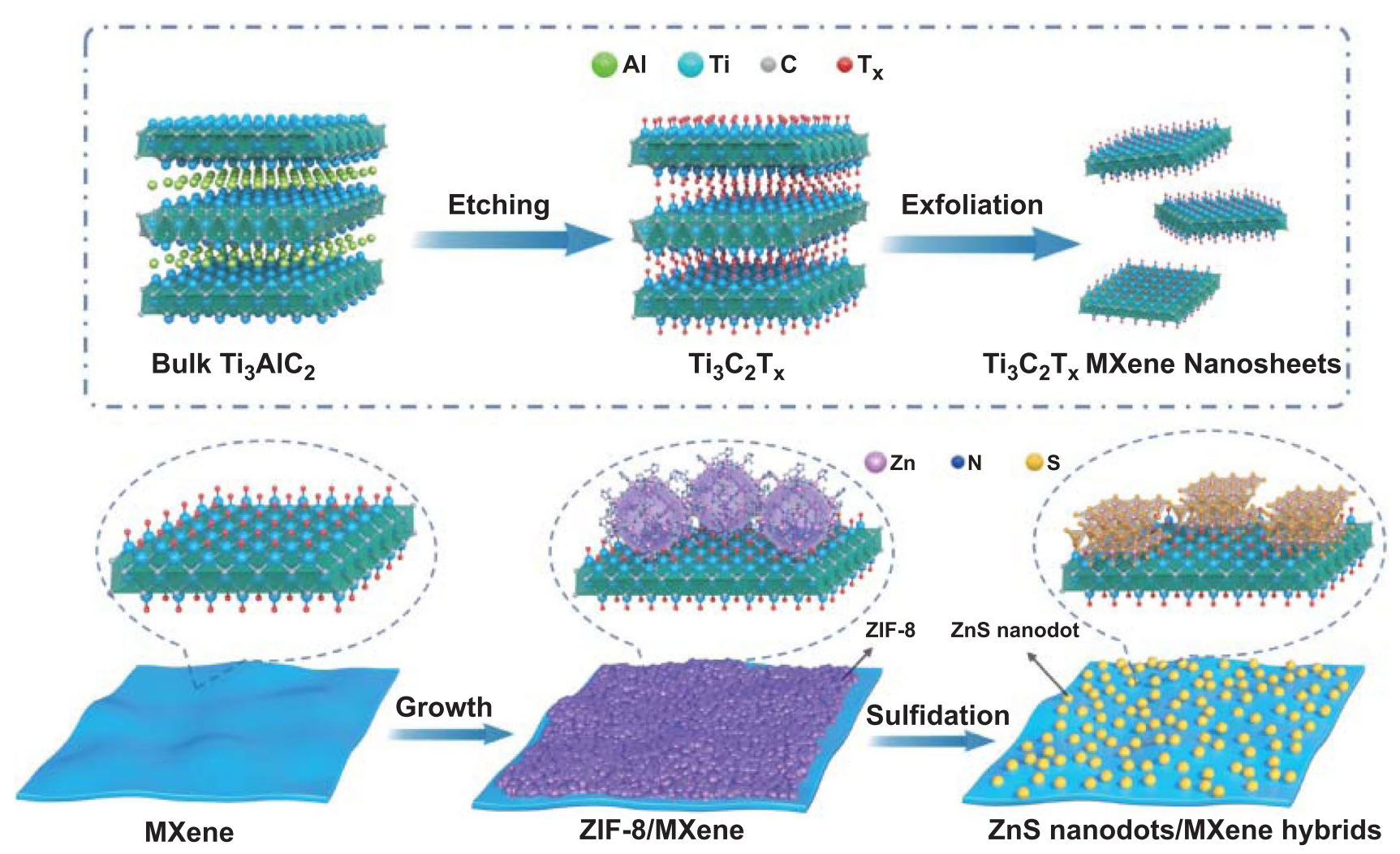

Fig. 1 Schematic illustration for the synthesis of ZnS/MXene hybrids 
formation of ZIF-8/MXene hybrids. The subsequent sulfidation process was completed in methanol/water mixture with TAA under refluxing at $60{ }^{\circ} \mathrm{C}$ under a nitrogen atmosphere. The weak coordination bond between 2-MeIM and $\mathrm{Zn}^{2+}$ ion was easily broken [13], and thus, the exposed $\mathrm{Zn}^{2+}$ can recombine with the $\mathrm{S}^{2-}$ produced by the TAA hydrolysis to form $\mathrm{ZnS}$.

The morphologies of ZIF-8, intermediate ZIF-8/MXene and the final product $\mathrm{ZnS}$ nanodots/MXene hybrids were characterized using SEM and TEM. SEM image (Fig. 2a) shows that the obtained ZIF-8 particles exhibit a typical rhombic dodecahedral shape with a homogeneous size of $\sim 300 \mathrm{~nm}$. In comparison with pure ZIF-8, ZIF-8 particles stabilized on the surface of MXene nanosheets exhibit smaller particle size of $\sim 50 \mathrm{~nm}$ (Figs. 2b, c and S3a) in ZIF-8/MXene-0.9. With the decrease in the MXene content, the ZIF-8 particles in ZIF-8/MXene-2 accumulate excessively on the surface of the MXene nanosheets (Fig. S3b, c). In addition, the particle size of ZIF-8 in ZIF-8/MXene-2 is $\sim 100 \mathrm{~nm}$, which is bigger and closer to rhombic dodecahedral shape than that in ZIF-8/MXene-0.9. The above results indicate that the $\mathrm{MXene}$ nanosheets can act as the nucleation sites for the growth of ZIF-8.

Figure 2d shows that ZnSMX64 has open interconnected structure and the surface of MXene nanosheets is rough. It can be seen that the small particles are uniformly distributed on the surface of MXene nanosheets. Energy-dispersive spectroscopy (EDS) elemental mapping (Fig. 2e)
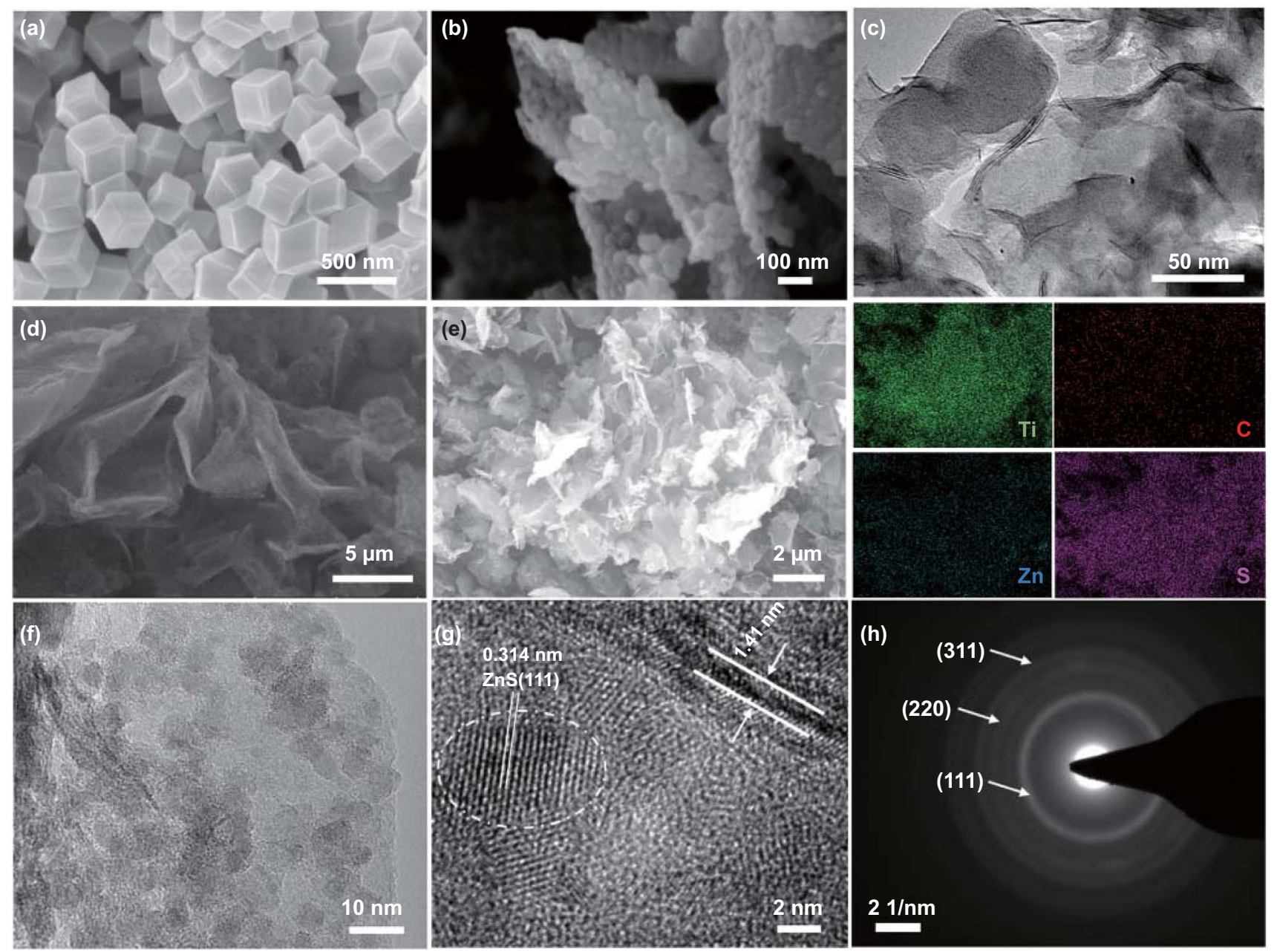

Fig. 2 Morphology characterizations. SEM images of a ZIF-8 and b ZIF-8/MXene-0.9, and $\mathbf{c}$ TEM image of ZIF-8/MXene-0.9. d SEM image of ZnS/MX64 and e EDS elemental mapping of Ti, S, Zn and C of the corresponding SEM image of the ZnS/MX64. f, $\mathbf{g}$ HRTEM images of ZnS/MX64 and $\mathbf{h}$ the corresponding SAED pattern 
of ZnSMX64 shows the uniform distribution of $\mathrm{Zn}, \mathrm{S}$, Ti, and $\mathrm{C}$ elements throughout the samples, suggesting the rough surface of the MXene nanosheet is composed of a large number of $\mathrm{ZnS}$ nanodots. HRTEM images (Fig. 2f, g) indicate that $\mathrm{ZnS}$ nanodots with particle sizes of 3-8 nm are uniformly and tightly anchored on the surface of 2D MXene nanosheets to form the 0D-2D structure. The lattice fringes with a d-spacing of 0.314 and $1.41 \mathrm{~nm}$ correspond to the (111) and (002) planes of $\mathrm{ZnS}$ [13] and MXene nanosheets [43] in the ZnSMX64, respectively. In addition, the hybridized boundary between $\mathrm{ZnS}$ nanodots and MXene nanosheets is a typical heterointerface structure, which may provide highly active sites for lithium adsorption and electrons transfer [6]. The corresponding selected area electron diffraction (SAED) pattern of ZnSMX64 in Fig. 2h displays dispersive diffraction rings related to (111), (220), (311) planes of $\mathrm{ZnS}$. For ZnSMX80 (Fig. S4a-c), the ZnS nanoparticles agglomerate severely on the surface of MXene due to the higher mass loading of $\mathrm{ZnS}$.

XRD was used to characterize the crystallinity and phase component of the samples. The XRD patterns of ZIF-8 (Fig. S5a) and MXene (Fig. 3a) are well agreed with previous reports $[13,30]$. In diffraction pattern of MXene, the strong peak at $6.8^{\circ}$ corresponds to (002) peak of $\mathrm{Ti}_{3} \mathrm{C}_{2} \mathrm{~T}_{x}$ MXene film [44]. The XRD patterns of the ZIF-8/MXene-0.9 and ZIF-8/ MXene-2 show characteristic peaks of ZIF-8 and MXene (Fig. $\mathrm{S} 5 \mathrm{~b}, \mathrm{c}$ ). However, after sulfidation, the XRD patterns (Fig. 3a) of ZnSMX64 and ZnSMX80 exhibit the characteristic peaks of $\mathrm{ZnS}$ (PDF No. 05-0566) and MXene, in which three diffraction peaks at $2 \theta$ of $28.6^{\circ}, 47.5^{\circ}$, and $56.3^{\circ}$ are assigned to (111), (220), and (311) planes of cubic structure $\mathrm{ZnS}$ [13, 45 ] and the peak round $6.2^{\circ}$ is the characteristic (002) peak of MXene. This result indicates the coexistence of $\mathrm{ZnS}$ and MXene nanosheets after the sulfidation. Besides, the $d_{002}$ of MXene in the ZnS/MXene hybrids increase to $14.1 \AA$ for ZnSMX64 and 14.3 $\AA$ for ZnSMX80, larger than that of pure MXene (13 $\AA$ ), which may be ascribed to the existence of methanol molecules in MXene nanosheets.

Raman spectra of the samples are presented in Figs. $3 \mathrm{~b}$ and S6. The Raman spectrum of MXene shows peaks around $200,387,622$, and $708 \mathrm{~cm}^{-1}$, corresponding to the typical characteristic vibrational modes for $\mathrm{Ti}_{3} \mathrm{C}_{2} \mathrm{~T}_{x}$ MXene [46, 47]. As shown in Fig. S6, Raman spectra of ZIF-8/ MXene composites mainly exhibit the characteristics of ZIF-8, which is ascribed to ZIF-8 anchoring on the surface of MXene nanosheets. For the ZnS/MXene hybrids, the peak at $285 \mathrm{~cm}^{-1}$ is assigned to $\mathrm{ZnS}[48,49]$. Thus, the main characteristic peaks of ZnS/MXene hybrids (Fig. 3b) can be ascribed to MXene and $\mathrm{ZnS}$ species. Moreover, the characteristic peak $\left(708 \mathrm{~cm}^{-1}\right)$ representing the vibration of the $\mathrm{C}$ atoms in $\mathrm{Ti}_{3} \mathrm{C}_{2} \mathrm{O}(\mathrm{OH})$ [46] vanishes in the $\mathrm{ZnS}$ / MXene hybrids, implying that the surface of $\mathrm{Ti}_{3} \mathrm{C}_{2} \mathrm{~T}_{x}$ MXene was hybridized with $\mathrm{ZnS}$ nanodots by dehydrogenation of hydroxyl functional group.

According to the XPS survey spectra, $\mathrm{Zn}$ and $\mathrm{S}$ peaks related to $\mathrm{ZnS}$ nanodots are clearly detected in the $\mathrm{ZnS} /$ MXene hybrids in contrast with pure $\mathrm{Ti}_{3} \mathrm{C}_{2} \mathrm{~T}_{x}$ MXene (Fig. $\mathrm{S} 7)$. The high-resolution $\mathrm{C} 1 s$ spectrum of pure MXene (Fig. S8a) is fitted by three peaks at $281.8 \mathrm{eV}$ (C-Ti), $285 \mathrm{eV}(\mathrm{C}-\mathrm{C} / \mathrm{C}=\mathrm{C})$, and $286.2 \mathrm{eV}(\mathrm{C}-\mathrm{O})$ [50], while for that of $\mathrm{ZnS} / \mathrm{MXene}$ hybrids (Fig. S8b, c), it is evident that the $\mathrm{C}-\mathrm{Ti}$ bond $(281.8 \mathrm{eV})$ is originated from $\mathrm{Ti}_{3} \mathrm{C}_{2} \mathrm{~T}_{x}$ MXene. The high-resolution Ti $2 p$ XPS spectra of pristine $\mathrm{Ti}_{3} \mathrm{C}_{2} \mathrm{~T}_{x}$ MXene (Fig. 3c) show multiple peaks as $\mathrm{Ti}-\mathrm{C}$ $\left(2 p_{3 / 2} 455.2 \mathrm{eV}\right.$ and $\left.2 p_{1 / 2} 458.8 \mathrm{eV}\right), \mathrm{Ti}^{2+}\left(2 p_{3 / 2} 455.8 \mathrm{eV}\right.$ and $\left.2 p_{1 / 2} 461.4 \mathrm{eV}\right)$, and $\mathrm{Ti}^{3+}\left(2 p_{3 / 2} 457.0 \mathrm{eV}\right.$ and $2 p_{1 / 2} 462.8$ $4 \mathrm{~V}$ ), which are consistent with the typical values reported for $\mathrm{Ti}_{3} \mathrm{C}_{2} \mathrm{~T}_{x}$ MXene $[1,51]$. The high-resolution Ti $2 p$ XPS spectra of ZnS/MXene hybrids (Figs. 3d and S9) are similar with that of MXene, confirming the stability of MXene in $\mathrm{ZnS} / \mathrm{MXene}$ hybrids without oxidation. Moreover, the interfaces interaction state of MXene and $\mathrm{ZnS}$ can be further investigated in O $1 s$ XPS spectra. As shown in Fig. $\mathrm{S} 10 \mathrm{a}$, the $\mathrm{O} 1 \mathrm{~s}$ region of MXene can be fitted into two peaks located at $530 \mathrm{eV}(\mathrm{Ti}-\mathrm{O})$ and $531.5 \mathrm{eV}(\mathrm{Ti}-\mathrm{OH})$ [39]. It is noteworthy that there is a new fitted peak at $532.3 \mathrm{eV}$ in the $\mathrm{ZnS} / \mathrm{MXene}$ hybrids, which may be attributed to Ti-O-Zn bonds [52] (Figs. 3e and S10b), indicating the formation of chemical bonds at the interface between $\mathrm{ZnS}$ and MXene. The formation of the Ti-O-Zn interfacial bonding is helpful to anchor $\mathrm{ZnS}$ nanodots on the MXene matrix tightly as well as strengthen the interaction between the two components.

The high-resolution $\mathrm{Zn} 2 p$ spectra of the $\mathrm{ZnS} / \mathrm{MXene}$ hybrids and $\mathrm{ZnS}$ are divided into one couple of peaks (Fig. 3f): the $2 p_{1 / 2}$ and $2 p_{3 / 2}$, which confirms the existence of $\mathrm{Zn}$ in the composites with the formation of $\mathrm{Zn}^{2+}$ [53]. Significantly, compared to pure $\mathrm{ZnS}$, both the $\mathrm{Zn}$ $2 p_{1 / 2}$ and $\mathrm{Zn} 2 p_{3 / 2}$ peaks of the $\mathrm{ZnS} / \mathrm{MX}$ ene hybrids shift to lower binding energy. It suggests that a more electron-rich state of $\mathrm{Zn} 2 p$ in the $\mathrm{ZnS} / \mathrm{MXene}$ hybrids than that of pure $\mathrm{ZnS}$, which may be attributed to the electron transfer from MXene to $\mathrm{ZnS}$ nanodots. In addition, 

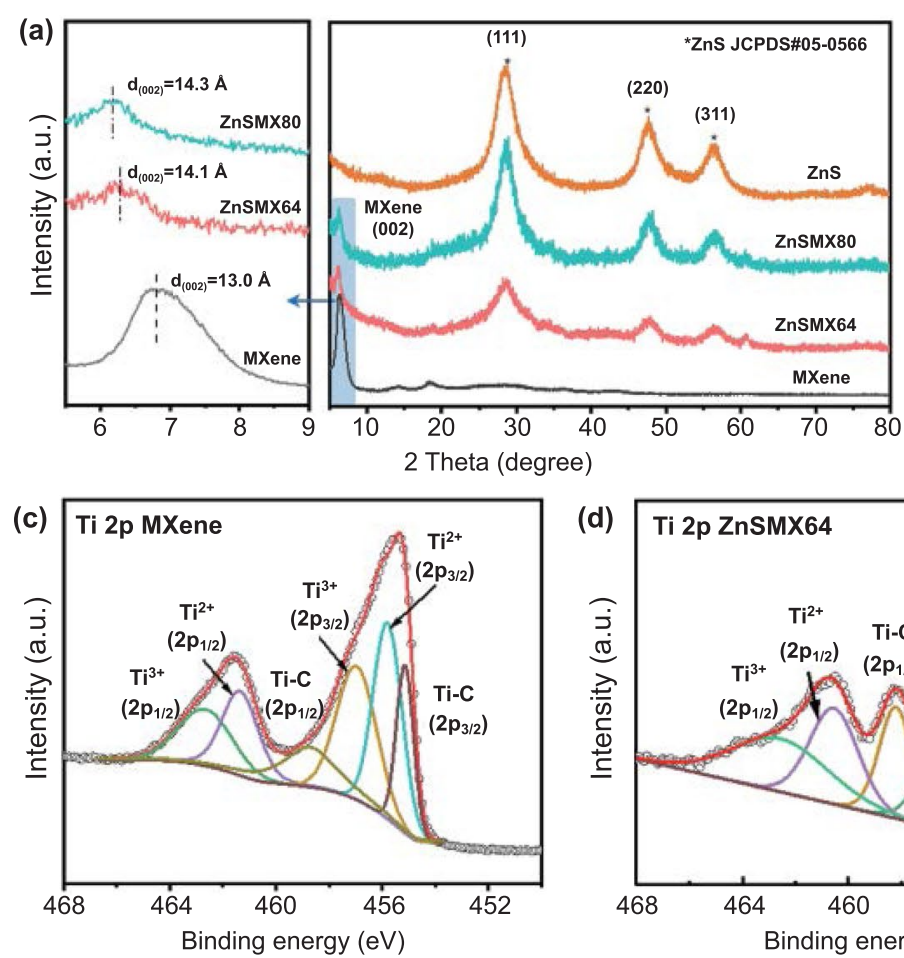
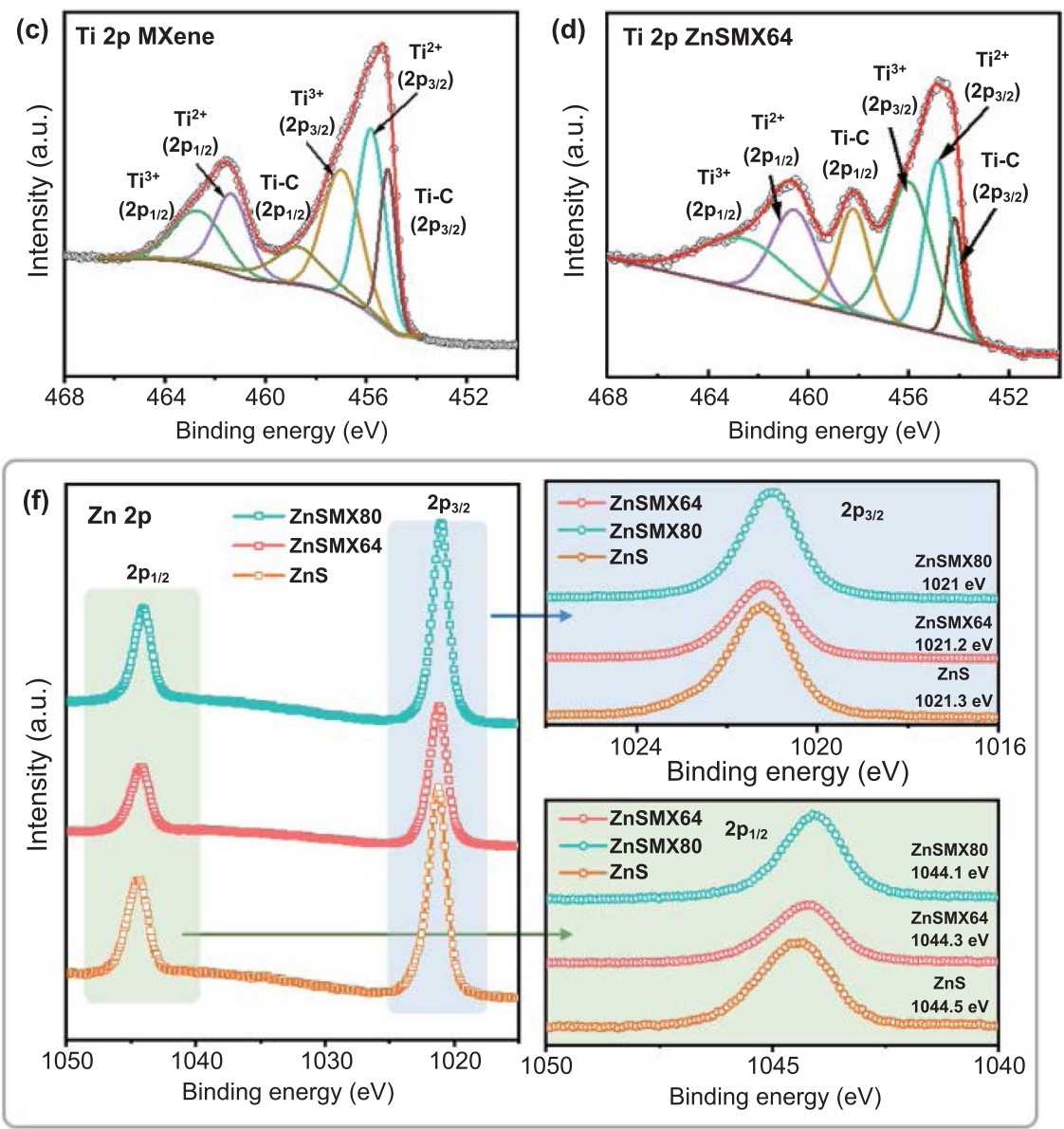
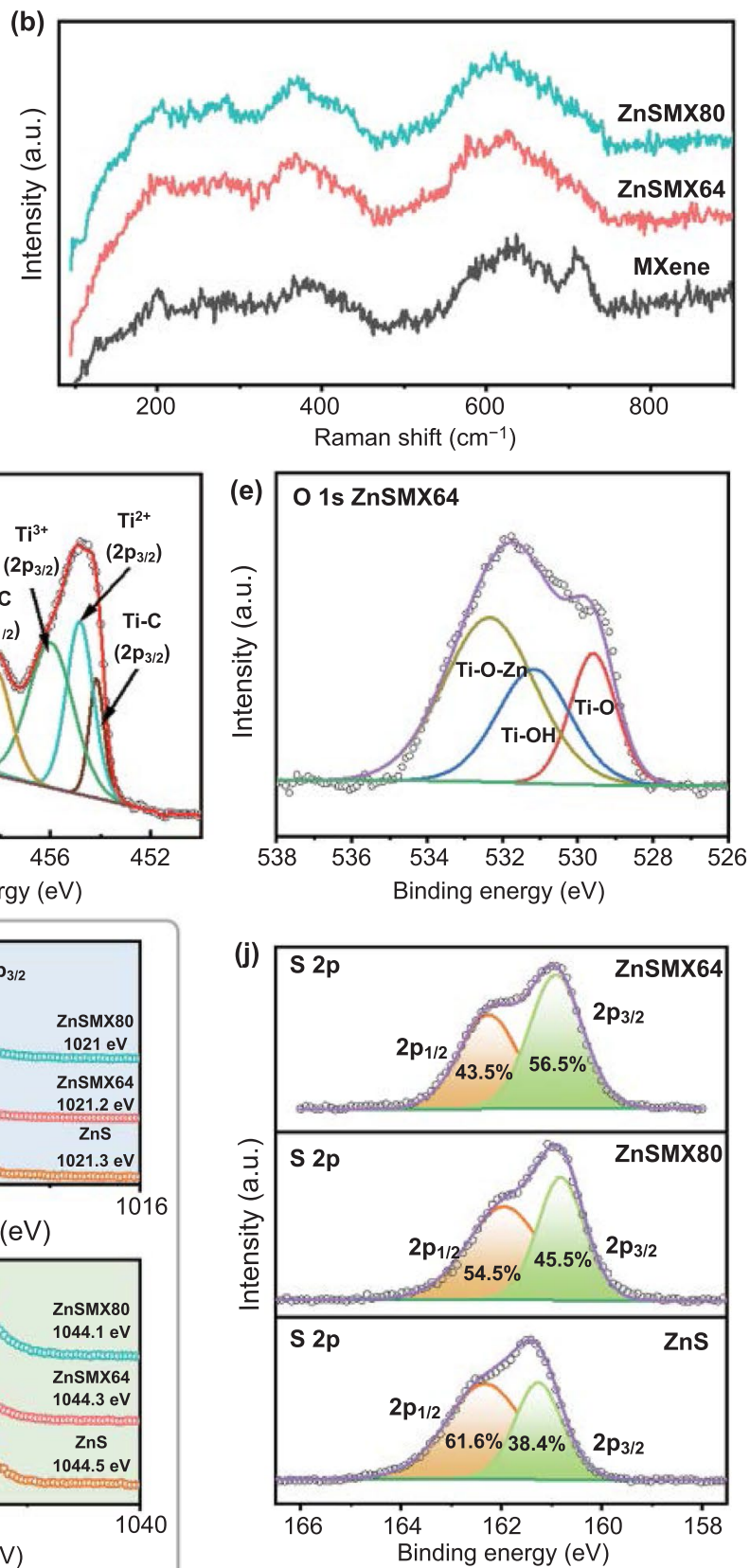

Fig. 3 Structural characterizations of the ZnS nanodots/MXene hybrids. a XRD patterns, b Raman spectra, and $\mathbf{c}$, $\mathbf{d}$ high-resolution Ti $2 p$, e O $1 s, \mathbf{f} \mathrm{Zn} 2 p, \mathbf{j}$ S $2 p$ XPS spectra

as shown in Fig. 3j, the electron redistribution between $\mathrm{ZnS}$ nanodots and MXene nanosheets is further confirmed by the $\mathrm{S} 2 p$ spectra with the characteristic peaks of $2 p_{3 / 2}$ and $2 p_{1 / 2}$ peaks in the $\mathrm{ZnS}$ phase [54]. Clearly, compared

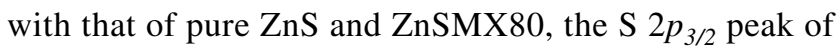
ZnSMX64 shifts to a lower binding energy with increased content, further proving the electron accumulation at $\mathrm{ZnS}$ nanodots [55]. Therefore, the above results demonstrate that the strong interfacial interactions with Ti-O-Zn bonds formed at the interfaces between $\mathrm{ZnS}$ nanodots and MXene nanosheets promotes the interface electron transfer, which are expected to significantly improve the electrochemical performance of the $\mathrm{ZnS}$ nanodots/MXene hybrids. 
The specific surface areas and pore structures of $\mathrm{ZnS}$, MXene and ZnS/MXene hybrids were studied by their $\mathrm{N}_{2}$ $(77 \mathrm{~K})$ adsorption/desorption isotherms. The isotherms of $\mathrm{ZnS}$ and ZnS/MXene hybrids exhibited in Fig. S11a are similar to typical IV hysteresis loops as defined by IUPAC $[21,56]$. It can be seen that the specific surface areas of the $\mathrm{ZnS} / \mathrm{MX}$ ene hybrids $\left(161.3 \mathrm{~m}^{2} \mathrm{~g}^{-1}\right.$ for $\mathrm{ZnSMX64}$, $153.4 \mathrm{~m}^{2} \mathrm{~g}^{-1}$ for $\mathrm{ZnSMX80)}$ are higher than those of the $\mathrm{ZnS}\left(141.2 \mathrm{~m}^{2} \mathrm{~g}^{-1}\right)$ and MXene $\left(19.0 \mathrm{~m}^{2} \mathrm{~g}^{-1}\right)$. Moreover, the pore volume of ZnSMX64 $\left(0.163 \mathrm{~cm}^{3} \mathrm{~g}^{-1}\right)$ and ZnSMX80 $\left(0.145 \mathrm{~cm}^{3} \mathrm{~g}^{-1}\right)$ are much higher than those of $\mathrm{ZnS}\left(0.109 \mathrm{~cm}^{3} \mathrm{~g}^{-1}\right)$ and MXene $\left(0.078 \mathrm{~cm}^{3} \mathrm{~g}^{-1}\right)$. The larger surface area and pore volume of $\mathrm{ZnS} / \mathrm{MX}$ ene hybrids are expected to synergistically promote fast ion diffusion and accommodate substantial volume changes. Figure S11b shows the pore size distributions of the $\mathrm{ZnS}$, MXene and $\mathrm{ZnS} / \mathrm{MXene}$ hybrids. The abundant mesopores $(2-10 \mathrm{~nm})$ are observed in the $\mathrm{ZnS} / \mathrm{MXene}$ hybrids, which is beneficial for facilitating the transport of $\mathrm{Li}^{+}$, thus improving their rate performance.

In order to shed light on the phase evolution of $\mathrm{ZnS}$ during lithium storage, the in situ XRD characterization was carried out for commercial $\mathrm{ZnS}(\mathrm{CZnS})$ anodes. In comparison with the XRD patterns of the as-prepared $\mathrm{ZnS} / \mathrm{MXene}$ samples, the XRD pattern (Fig. S12) of CZnS shows a similar crystal structure but larger crystal size. The electrochemical behavior of CZnS was also evaluated by CV (Fig. S13), which shows undesirable electrochemical reversibility similar to that of $\mathrm{ZnS}$ prepared in this work. The phase evolution of $\mathrm{CZnS}$ during the first lithium storage cycle was shown in the contour plot with corresponding voltage profiles (Fig. 4a) and diffraction patterns (Fig. 4b). The intensity of characteristic peaks of $\mathrm{ZnS}$ gradually decreases upon lithiation due to the conversion reaction of $\mathrm{ZnS}$ with lithium. Subsequently, the existence of $\mathrm{Li}_{2} \mathrm{~S}$ is confirmed by the emergence of (111) peak of $\mathrm{Li}_{2} \mathrm{~S}$ while $\mathrm{Zn}$ metal and $\mathrm{Li}_{x} \mathrm{Zn}$ are not observed. At full lithiation state, both $\mathrm{LiZn}$ and $\mathrm{Li}_{2} \mathrm{~S}$ are observed as final products. During delithiation, the characteristic peaks of $\mathrm{LiZn}$ gradually disappear until the emergence of $\mathrm{Li}_{\mathrm{x}} \mathrm{Zn}$ (such as $\mathrm{LiZn}_{4}$ ) [57]. Immediately, the phase of $\mathrm{Li}_{x} \mathrm{Zn}$ vanishes while the phase of $\mathrm{Zn}$ metal appeares. The intensity of (101) peak of $\mathrm{Zn}$ firstly exhibits increases due to the accumulation of $\mathrm{Zn}$ metal, as lithium is totally extracted from Li-Zn alloy. Subsequently, the intensity of (101) peak of Zn gradually decreases upon delithiation due to the consumption of $\mathrm{Zn}$ in the conversion reaction. At full delithiation
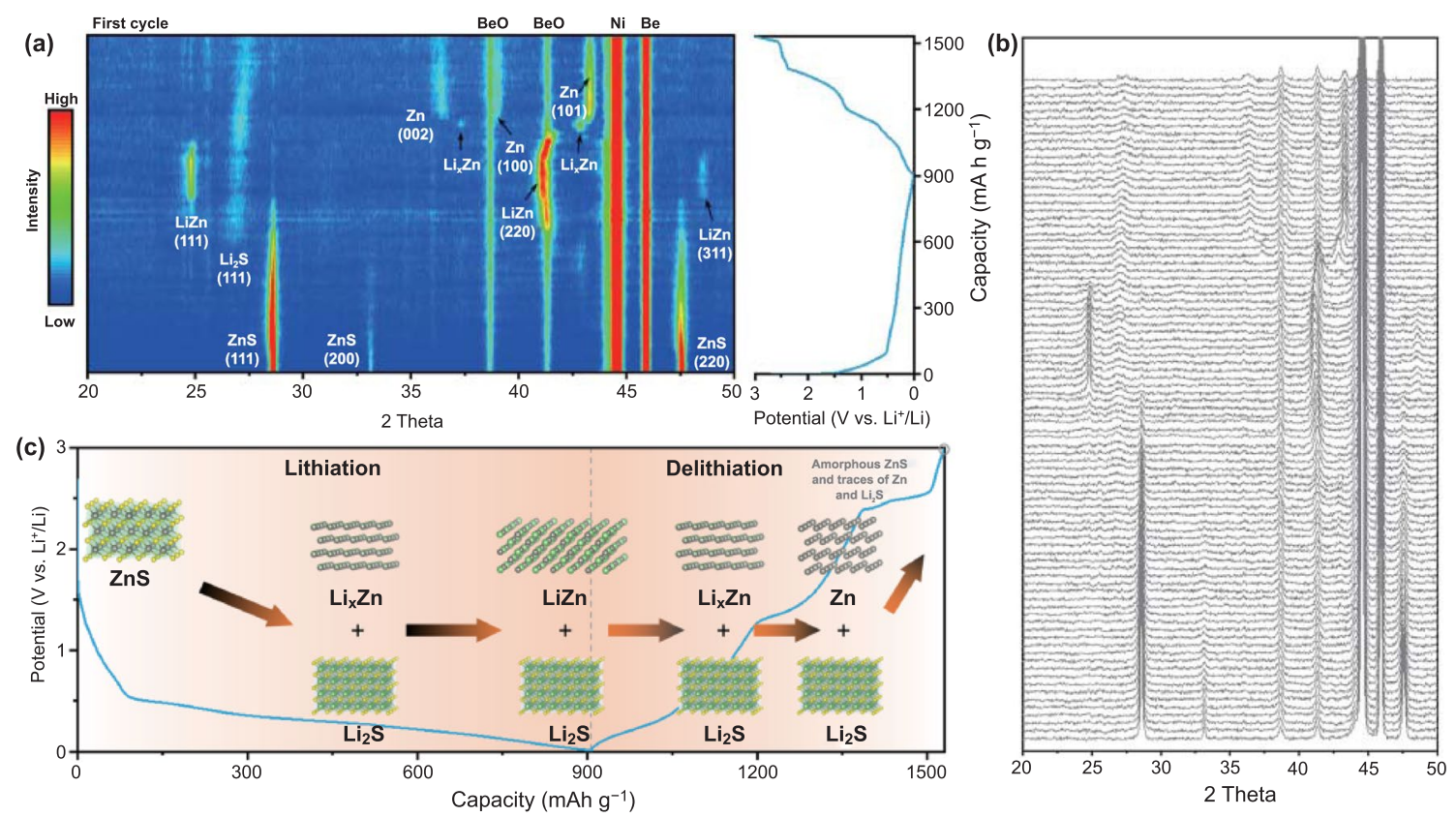

Fig. 4 In situ XRD characterization of commercial ZnS anodes during lithium storage. a Contour plot of in situ XRD characterization with corresponding voltage profile and $\mathbf{b}$ diffraction patterns for $\mathrm{CZnS}$ anode in the first cycle. $\mathbf{c}$ Schematic illustration of the phase evolution of the $\mathrm{CZnS}$ in the first lithiation-delithiation cycle 
state, $\mathrm{ZnS}$ phase cannot be observed, while the weak peaks of $\mathrm{Zn}$ and $\mathrm{Li}_{2} \mathrm{~S}$ still remain, which is ascribed to the amorphous nature of the new generated $\mathrm{ZnS}$ and the poor reversibility of the conversion reaction, respectively [57]. Based on the above results, the phase evolution of $\mathrm{ZnS}$ during first lithiation-delithiation cycle is illustrated in (Fig. 4c). In the second cycle, the phase evolution is also shown in the contour plot (Fig. S14) and the corresponding diffraction patterns (Fig. S15). During lithiation, the amorphous ZnS is firstly converted to $\mathrm{Zn}$ and $\mathrm{Li}_{2} \mathrm{~S}$, leading to the increasing intensity of characteristic peak of $\mathrm{Zn}$. Afterward, $\mathrm{Li}_{\mathrm{x}} \mathrm{Zn}$ was formed during further lithiation due to the alloying reaction of $\mathrm{Zn}$ with lithium. At full lithiation state, the LiZn phase is finally formed, but its peak intensity is weaker than that in the first cycle, suggesting poor lithium storage reversibility. During delithiation, a reverse process is observed. According to the above results, the following lithium storage mechanism of $\mathrm{ZnS}$ is proposed.

First cycle: $\mathrm{ZnS} \rightarrow$ amorphous $\mathrm{Li}_{x} \mathrm{Zn}+\mathrm{Li}_{2} \mathrm{~S} \rightarrow \mathrm{LiZn}+\mathrm{Li}_{2} \mathrm{~S}$ (full lithiation state) $\rightarrow \mathrm{Li}_{x} \mathrm{Zn}+\mathrm{Li}_{2} \mathrm{~S} \rightarrow \mathrm{Zn}+\mathrm{Li}_{2} \mathrm{~S} \rightarrow$ amorphous $\mathrm{ZnS}+$ traces of $\mathrm{Zn}$ and $\mathrm{Li}_{2} \mathrm{~S}$.

Second cycle: amorphous $\mathrm{ZnS}+$ traces of $\mathrm{Zn}$ and $\mathrm{Li}_{2} \mathrm{~S} \rightarrow \mathrm{Zn}+\mathrm{Li}_{2} \mathrm{~S} \rightarrow \mathrm{Li}_{x} \mathrm{Zn}+\mathrm{Li}_{2} \mathrm{~S} \rightarrow \mathrm{LiZn}+\mathrm{Li}_{2} \mathrm{~S}$ (full lithiation state) $\rightarrow \mathrm{Li}_{\mathrm{x}} \mathrm{Zn}+\mathrm{Li}_{2} \mathrm{~S} \rightarrow \mathrm{Zn}+\mathrm{Li}_{2} \mathrm{~S} \rightarrow$ amorphous $\mathrm{ZnS}+$ traces of $\mathrm{Zn}$ and $\mathrm{Li}_{2} \mathrm{~S}$.

Based on the above analysis, the lithium storage mechanism of CZnS can be simply described as following: the $\mathrm{ZnS}$ is converted into $\mathrm{LiZn}$ and $\mathrm{Li}_{2} \mathrm{~S}$ during lithiation; however, the resultant $\mathrm{LiZn}$ and $\mathrm{Li}_{2} \mathrm{~S}$ incompletely recombine in delithiation, causing the presence of traces of $\mathrm{Zn}$ and $\mathrm{Li}_{2} \mathrm{~S}$, which reveal the poor reversibility of $\mathrm{ZnS}$ during lithium storage. In the second cycle, the irreversible phase transition occurs in $\mathrm{ZnS}$ more severely. The poor reversibility is the main reason for the quick capacity decay during cycling for $\mathrm{CZnS}$ (Fig. S16), which should be ascribed to the large volume expansion of $\mathrm{ZnS}$ as well as the consequent electrode pulverization (Fig. S17). Therefore, in order to overcome this problem and improve its cycle life, $\mathrm{ZnS}$ nanodots were prepared and further in situ riveted on MXene substrate to improve the lithium storage reversibility.

The electrochemical behaviors of the $\mathrm{ZnS}$ and $\mathrm{ZnS} /$ MXene hybrids were evaluated by $\mathrm{CV}$ and galvanostatic charge-discharge test. The $\mathrm{CV}$ curves of pure $\mathrm{ZnS}$, ZnSMX64, and ZnSMX80 at a scan rate of $0.1 \mathrm{mV} \mathrm{s}^{-1}$ are shown in Figs. 5a, b and S18. It can be seen that the pure
$\mathrm{ZnS}$ and $\mathrm{ZnS} / \mathrm{MXene}$ hybrids show similar electrochemical behaviors in the first cycle, but the redox peaks in the following cycles for the pure $\mathrm{ZnS}$ are quite different from those in the first cycle due to the low reversibility. From the CV curves of the ZnS/MXene hybrids (Figs. 5b and $\mathrm{S} 18$ ), we can perceive that the incorporation of MXene with $\mathrm{ZnS}$ nanodots can greatly improve the lithium storage reversibility. In particular, when introducing the appropriate amount of MXene, the CV curves of ZnSMX64 almost overlap after the first cycle, manifesting the highly stable structure and reversible reaction behavior during lithium storage cycling. Figures S19 and 5c show the first three charge-discharge curves of $\mathrm{ZnS}$ and ZnSMX64 electrodes at $100 \mathrm{~mA} \mathrm{~g}^{-1}$. Pure $\mathrm{ZnS}$ shows a lithiation/delithiation capacity of $1141.5 / 720.9 \mathrm{mAh} \mathrm{g}^{-1}$ with an initial Coulombic efficiency (ICE) of 63.2\%. However, the gradual separated voltage profile from 1st to 4th cycle indicates unstable cyclability. The MXene delivers an initial lithiation/delithiation capacity of 355.3/166.3 $\mathrm{mAh} \mathrm{g}^{-1}$, and no obvious voltage platform appears in the voltage range of $0.01-2.5 \mathrm{~V}$ (Fig. S20). For the ZnSMX64, the initial lithiation/delithiation capacity is $1246.9 / 683.2 \mathrm{mAh} \mathrm{g}^{-1}$ with the corresponding ICE of $54.8 \%$. The higher capacity of ZnS/MXene hybrids than that of MXene is ascribed to the capacity contribution from $\mathrm{ZnS}$ nanodots. The comparison of the cycling performance of MXene, $\mathrm{ZnS}$ and $\mathrm{ZnS} / \mathrm{MXene}$ hybrids at $100 \mathrm{~mA} \mathrm{~g}^{-1}$ is shown in Fig. 5d. For pure $\mathrm{ZnS}$, the lithiation capacity decreases sharply to $190 \mathrm{mAh} \mathrm{g}^{-1}$ after 20 cycles. The rapid capacity fading may be ascribed to the pulverization of $\mathrm{ZnS}$ due to its large volume change during charge-discharge [58]. In contrast, the ZnSMX64 exhibits the best cycle stability and a high capacity of $650.6 \mathrm{mAh} \mathrm{g}^{-1}$ is remained after 100 cycles. Figure S21 shows the voltage profiles of ZnSMX64 under different cycles at a current density of $100 \mathrm{~mA} \mathrm{~g}^{-1}$. The voltage profiles almost overlap even after 100 cycles. This result indicates that the introducing of MXene is powerful to improve the cyclability of the $\mathrm{ZnS} / \mathrm{MXene}$ hybrids electrodes, which agrees with the $\mathrm{CV}$ results mentioned above. It is noteworthy that ZnSMX64 exhibits better cyclability than ZnSMX80 anode, suggesting that the superfluous active $\mathrm{ZnS}$ will sacrifice its cycling performance. The insufficient MXene nanosheets in ZnSMX80 may not accommodate the substantial volume variation of agglomerated $\mathrm{ZnS}$ nanoparticles during lithium storage cycling. Moreover, the long-term cycling performance of ZnSMX64 at $0.5 \mathrm{~A} \mathrm{~g}^{-1}$ is shown in Fig. 5e. The ZnSMX64 

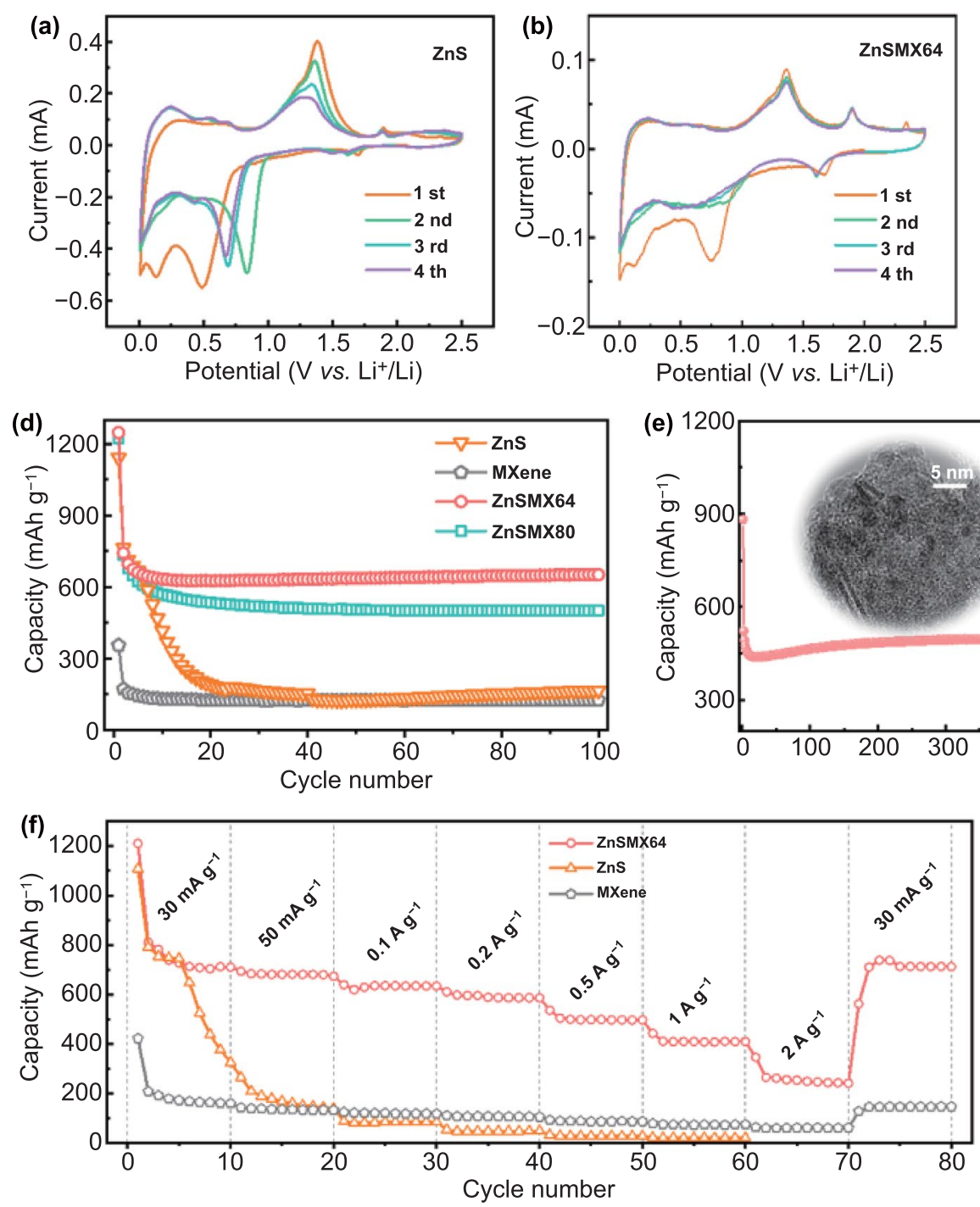
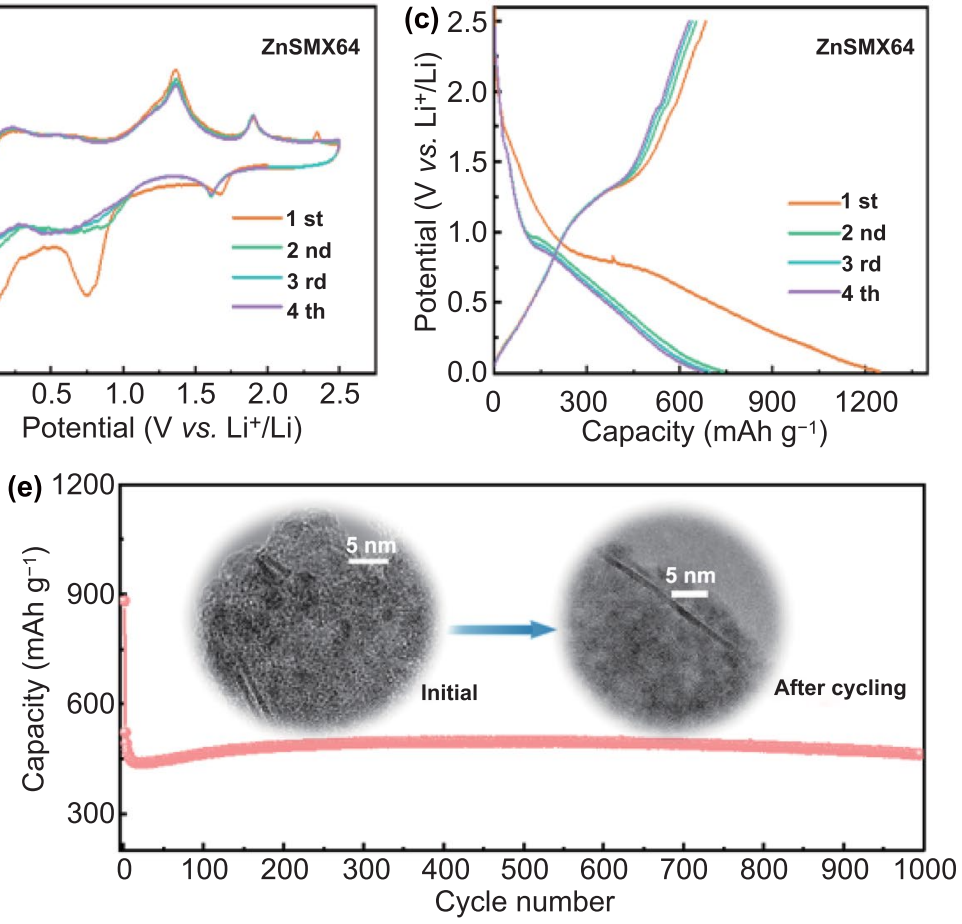

Fig. 5 Lithium storage performance. CV curves of as-prepared a $\mathrm{ZnS}$ and $\mathbf{b} \mathrm{ZnSMX64}$ at a scan rate of $0.1 \mathrm{mV} \mathrm{s}^{-1}$. $\mathbf{c}$ Galvanostatic charge/ discharge profiles of ZnSMX64 at $100 \mathrm{~mA} \mathrm{~g}^{-1}$. d Cyclability at $100 \mathrm{~mA} \mathrm{~g}^{-1}$, e long-term cycling performance of ZnSMX64 at $0.5 \mathrm{~A} \mathrm{~g}^{-1}$ with ex situ HRTEM images in the insets, $\mathbf{f}$ rate capabilities at different currents, and $\mathbf{g}$ Nyquist plots for $\mathrm{ZnS}$ and ZnS/MXene hybrids

exhibits a highly stable capacity of $462.8 \mathrm{mAh} \mathrm{g}^{-1}$ without obvious decay after 1000 cycles. From ex situ HRTEM characterization result, there is no structural collapse after 1000 cycles for ZnSMX64 electrode as shown in the insets of Fig. 5e. Therefore, such stable cyclability may be attributed to the rationally designed nanostructure of $\mathrm{ZnS}$ nanodots onto MXene substrate and the strong interfacial interaction between them.

Apart from the cycling performance, rate capability is also key factor to evaluate the potential value of the $\mathrm{ZnS} / \mathrm{MXene}$ hybrids as LIBs anodes. Figure $5 \mathrm{f}$ shows the rate capability of all the samples at different current densities. The pure
ZnS exhibits very poor rate performance with a capacity of $19.3 \mathrm{mAh} \mathrm{g}^{-1}$ at $1.0 \mathrm{~A} \mathrm{~g}^{-1}$. However, the ZnSMX64 anode exhibits excellent rate performance, especially under high current densities, which delivers reversible capacities of 726.8, 680.9, 635.1, 588.1, 497.7, 408.8, and $252.5 \mathrm{mAh} \mathrm{g}^{-1}$ at $0.03,0.05,0.1,0.2,0.5,1$, and $2 \mathrm{~A} \mathrm{~g}^{-1}$, respectively. Noticeably, a high capacity of $712.5 \mathrm{mAh} \mathrm{g}^{-1}$ can be retained when the current density is back to $0.03 \mathrm{~A} \mathrm{~g}^{-1}$. Moreover, EIS was used to investigate the charge transfer kinetics of the ZnS/MXene hybrids. As shown in Fig. 5g, the Nyquist plots consist of a semicircle in high and middle frequencies and a straight line at low frequency. The semicircle corresponds 
to the impedance of charge transfer, and the straight line is assigned to Li diffusion in electrodes [21]. Specifically, the Nyquist plot of MXene shows the smallest semicircle, indicating the lowest charge transfer resistance. In comparison with pure ZnS anode, both ZnSMX64 and ZnSMX80 show a smaller semicircle, indicating that the introducing MXene into $\mathrm{ZnS}$ nanodots can reduce charge transfer resistance and promote charge transfer kinetics. The semicircle of ZnSMX64 anodes is smaller than that of pure $\mathrm{ZnS}$ anode and ZnSMX80, suggesting the best charge transfer kinetics.

To explore the lithium storage behavior of the $\mathrm{ZnS} /$ MXene hybrids, $\mathrm{CV}$ curves were measured at different scan rates from 0.1 to $2 \mathrm{mV} \mathrm{s}^{-1}$ (Fig. 6a, b). Compared with ZnSMX80, ZnSMX64 has smaller redox potential differences $(0.6$ and $1.3 \mathrm{~V})$ at $2 \mathrm{mV} \mathrm{s}^{-1}$, indicating weaker polarization and enhanced electrochemical kinetics. Furthermore, the electrochemical kinetics of ZnSMX64 is analyzed by power-law relationship [59] between measured current and sweep rate according to Eqs. (1) and (2):

$i=a v^{b}$

$\log (i)=b \log (v)+\log (a)$
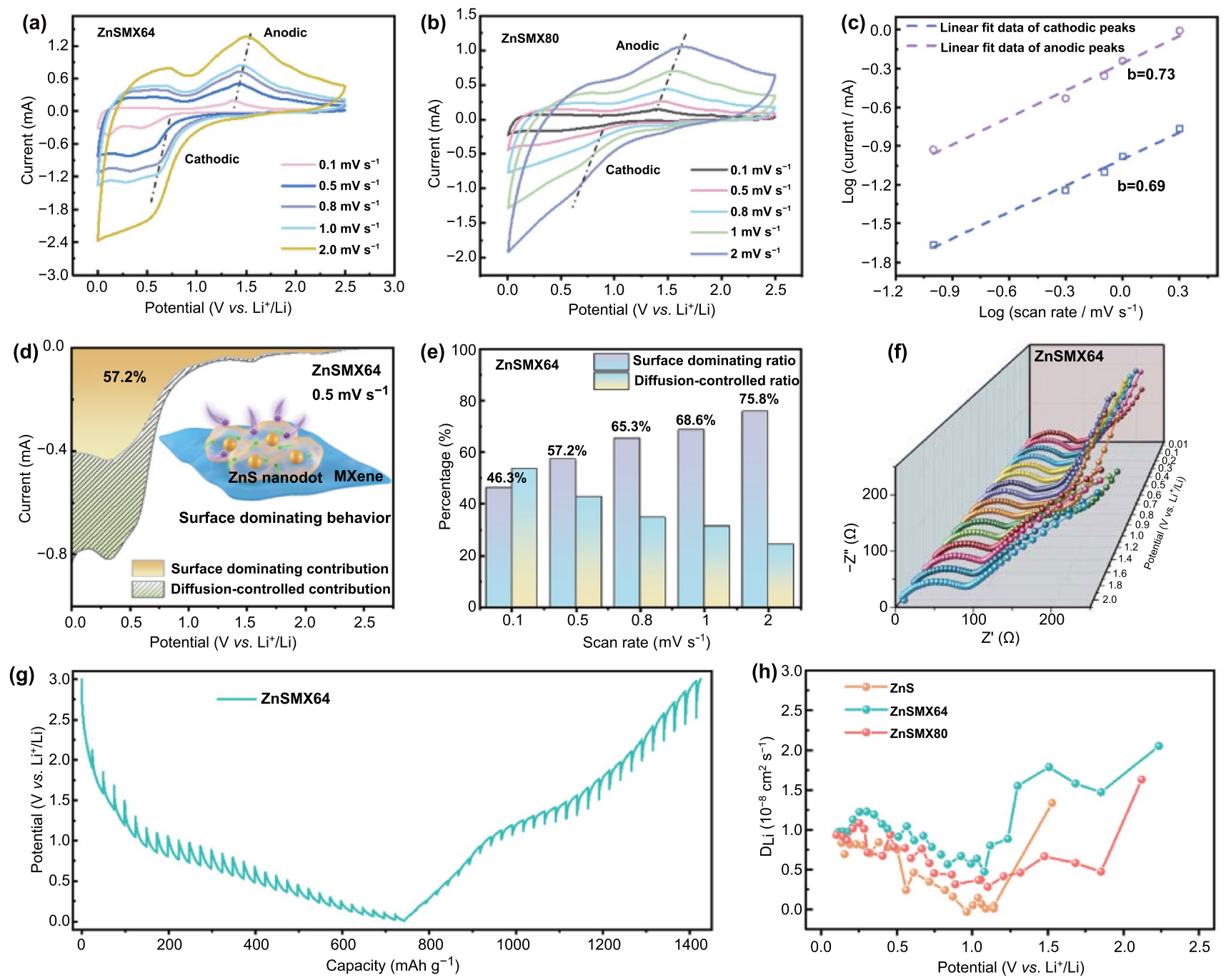

Fig. 6 Electrochemical kinetics for the ZnS/MXene hybrids. CV curves of a ZnSMX64 and b ZnSMX80 at different sweep rates from 0.1 to $2.0 \mathrm{~m} \mathrm{~V}^{-1}$, and $\mathbf{c}$ the plots of $\log i$ versus $\log v$. d CV curve of ZnSMX64 with the surface dominating capacity contribution for cathodic process at $0.5 \mathrm{mV} \mathrm{s}^{-1}$, and $\mathbf{e}$ the proportion of capacity contributions at different scan rates. $\mathbf{f}$ In situ EIS characterization of ZnSMX64 at different lithiation states between 2.0 and $0.01 \mathrm{~V}$. $\mathbf{g}$ GITT potential profile of the ZnSMX64 and $\mathbf{h}$ the $\mathrm{Li}^{+}$diffusion coefficients in lithiation process 
where $i$ is current, $a$ is a constant, $v$ is scan rate, and $b$ is the slope of the $\log (v)-\log (i)$ plot. The $b$ value close to 1 means a surface dominating behavior (non-diffusion-controlled process), while the $b$ value of 0.5 is a diffusion-controlled process. As shown in Fig. 6c, the value of $b$ is 0.69 and 0.73 for the cathodic and anodic peak of ZnSMX64, respectively, revealing non-ignored surface dominating lithium storage behavior with good electrochemical kinetics. Furthermore, the measured current can be analyzed with Eq. (3):

$i(v)=k_{1} v+k_{2} v^{1 / 2}$

where $k_{1}$ and $k_{2}$ are adjustable parameters, and $k_{1} v$ and $k_{2} v^{1 / 2}$ represent the surface dominating reaction current and diffusion-controlled reaction current, respectively [60]. As shown in Fig. 6d, the ZnSMX64 anode exhibits a surface dominating capacity contribution of $57.2 \%$ at a scan rate of $0.5 \mathrm{mV} \mathrm{s}^{-1}$, which is higher than that of ZnSMX80 (45.7\%) (Fig. S22a). In addition, it can be observed that surface dominating contribution ratios increased with scan rates for both ZnSMX64 (Fig. 6e) and ZnSMX80 (Fig. S22b). The higher surface dominating capacity contributions in ZnSMX64 are benefit to exhibit a superior rate capability [20].

In situ EIS was performed to further elucidate the impedance properties of the $\mathrm{ZnS} / \mathrm{MXene}$ hybrids with the $\mathrm{ZnS}$ anode as a comparison at different lithiation states (2.0-0.01 V). As shown in Figs. 6f and S22, the Nyquist plots exhibit gradually increased semicircle during the lithiation process, indicating increased charge transfer resistance. This result may be ascribed to the formation of SEI and gradually saturated lithiation state [6]. Moreover, the Nyquist plots of ZnSMX64 exhibit the smallest semicircle in the whole lithiation process compared with ZnSMX80 and $\mathrm{ZnS}$ anode, indicating the lowest charge transfer impedance and best charge transfer kinetics during lithiation. Furthermore, the galvanostatic intermittent titration technique (GITT) was used to investigate the $\mathrm{Li}^{+}$diffusion properties as shown in Figs. $6 \mathrm{~g}$ and $\mathrm{S} 24$ [61]. The apparent $\mathrm{Li}^{+}$diffusion coefficients are calculated by Eq. S1. As shown in Fig. 6h, ZnSMX64 exhibits the higher apparent $\mathrm{Li}^{+}$diffusion coefficients than $\mathrm{ZnS}$ and ZnSMX80, indicating the excellent electrochemical kinetics. In addition, the lithium storage performance of $\mathrm{ZnS} / \mathrm{MXene}$ hybrids electrodes may be further improved if using MXene film instead of $\mathrm{Cu}$ foil as a current collector [62]. Therefore, the superior electrochemical performance of ZnSMX64 can be ascribed to its surface dominating lithium storage behavior with high reversibility and fast lithium diffusion capability.
Furthermore, the lithium adsorption and diffusion features at the $\mathrm{ZnS} / \mathrm{MXene}$ heterointerface and the corresponding interfacial electronic structure were studied by density functional theory (DFT) calculations. First, three possible $\mathrm{ZnS}-\mathrm{Ti}_{3} \mathrm{C}_{2} \mathrm{~T}_{x}$ MXene heterointerface models with different $\mathrm{ZnS}$ orientation (Fig. S25a) were constructed, i.e., ZnS (111)-MXene, ZnS (101)-MXene, and ZnS (001)-MXene. Among them, the $\mathrm{ZnS}$ (111)-MXene heterointerface is the most stable one because of the strongest interfacial interaction $\left(E_{\text {Binding }}=-2.851 \mathrm{eV}\right)$ (Fig. S25b). Therefore, the $\mathrm{ZnS}$ (111)-MXene heterointerface model was selected to evaluate lithium adsorption/migration features. The lithium adsorption energies at different sites in $\mathrm{ZnS}$ (111), MXene and $\mathrm{ZnS}$ (111)-MXene heterointerface were calculated, and the results are shown in Figs. S26, S27 and 7a, respectively. The ZnS (111)-MXene heterointerface exhibits the highest lithium adsorption capability due to the lowest average adsorption energies. Thus, the ZnS (111)-MXene heterointerface with strong lithium adsorption capability may function as active sites for improving lithium storage capacity. The interfacial interaction between $\mathrm{ZnS}$ and MXene was further clarified by the charge density differences, the density of states (DOS), and the planar average potential charge density. As shown in Fig. 7b, the charge density at the $\mathrm{ZnS}$ (111)-MXene heterointerface exhibits an accumulating tendency at the surface of $\mathrm{ZnS}$, confirming a significant charge transfer phenomenon. According to the DOS plots of MXene to $\mathrm{ZnS}-\mathrm{MXene}$ heterogeneous structure (Fig. 7c-e), the electron density near Fermi lever increases for $\mathrm{ZnS}$ (111)MXene heterointerface compared with that of $\mathrm{ZnS}$. Moreover, the planar average charge density along the direction of $z$ axis of ZnS-MXene heterointerface is shown in Fig. 7f, which further proves the electron transfer from the electronrich region $\left(\mathrm{Ti}_{3} \mathrm{C}_{2} \mathrm{~T}_{x} \mathrm{MXene}\right)$ to the electron-deficient region $(\mathrm{ZnS})$. The interfacial electron migration from MXene to $\mathrm{ZnS}$ leads to interfacial charge redistribution and the corresponding formation of an interfacial electric field within the interfaces, which may effectively promote electron transfer and ion diffusion to achieve excellent electrochemical performance. Furthermore, lithium diffusion features were evaluated for MXene, ZnS (111) and ZnS (111)/MXene heterointerface. The lithium migration pathways from a hollow site to adjacent hollow sites are shown in Figs. $7 \mathrm{~g}$ and S28. The diffusion relative energy is plotted in Fig. $7 \mathrm{~h}$. The lithium diffusion energy barrier of ZnS (111)/MXene heterointerface 
(a)

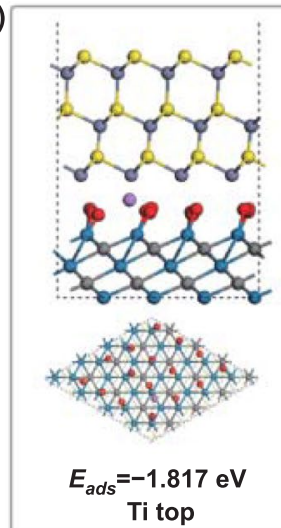

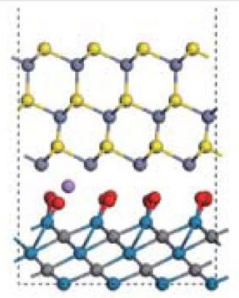

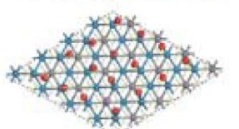

$E_{\text {ads }}=-0.795 \mathrm{eV}$

C top
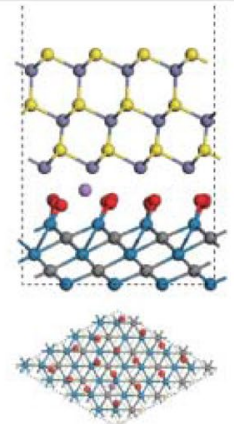

$E_{\text {ads }}=-1.265 \mathrm{eV}$ Ti-C top

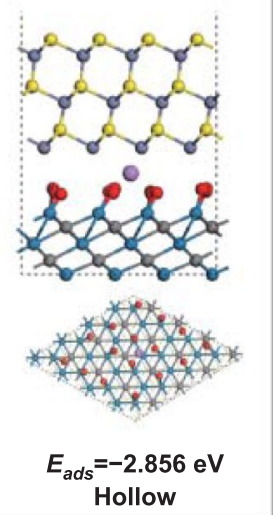

(b)
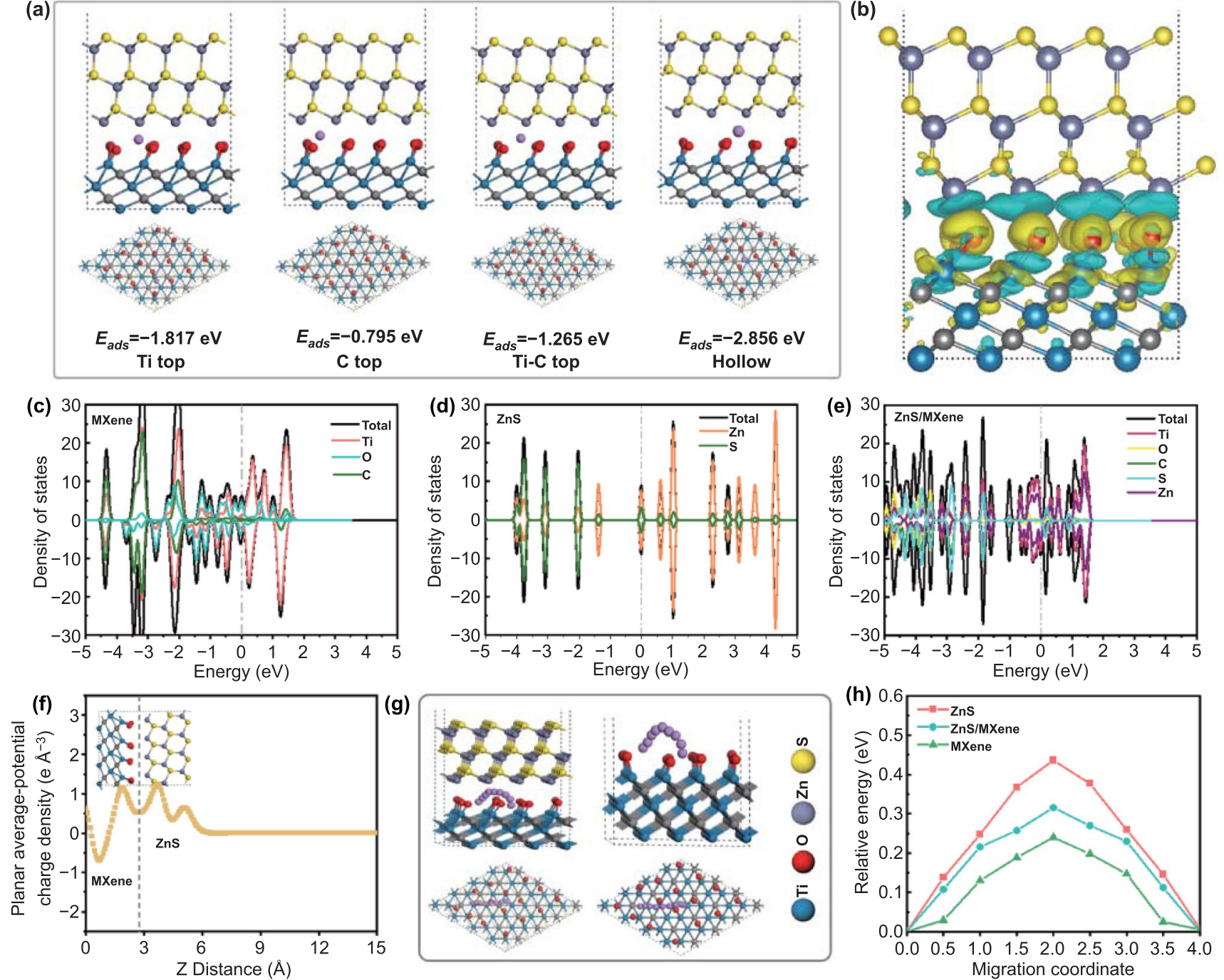

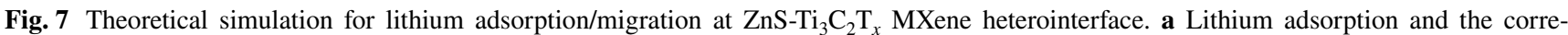
sponding adsorption energy at Ti top, $\mathrm{C}$ top, Ti-C top and hollow site at $\mathrm{ZnS}(111) / \mathrm{Ti}_{3} \mathrm{C}_{2} \mathrm{~T}_{x}$ MXene heterointerface and $\mathbf{b}$ charge density differences of $\mathrm{ZnS}(111) / \mathrm{Ti}_{3} \mathrm{C}_{2} \mathrm{~T}_{x}$ MXene heterointerface. DOS plots (fermi levels are set as zero and indicated with dashed lines) of $\mathbf{c} \mathrm{Ti}_{3} \mathrm{C}_{2} \mathrm{~T}_{x}, \mathbf{d ~ Z n S}$, and $\mathrm{e} \mathrm{ZnS} / \mathrm{Ti}_{3} \mathrm{C}_{2} \mathrm{~T}_{x}$ MXene. f Planar average potential charge density along the $\mathrm{z}$ axis (vertical direction) of $\mathrm{ZnS}(111) / \mathrm{Ti}_{3} \mathrm{C}_{2} \mathrm{~T}_{x} \mathrm{MXene}$ heterointerface. $\mathbf{g}$ Lithium diffusion pathway and $\mathbf{h}$ the corresponding relative diffusion energy variation at $\mathrm{ZnS}(111) / \mathrm{Ti}_{3} \mathrm{C}_{2} \mathrm{~T}_{x} \mathrm{MXene}$ heterointerface

$(0.32 \mathrm{eV})$ is lower than that of $\mathrm{ZnS}(0.44 \mathrm{eV})$, but higher than that of MXene $(0.24 \mathrm{eV})$. This result indicates that MXene in ZnS (111)/MXene heterointerface can promote fast lithium migration. In comparison with $\mathrm{ZnS}$, the lower diffusion energy barrier of $\mathrm{ZnS}$ (111)/MXene heterointerface enables fast lithium migration and excellent diffusion kinetics to boost rate performance. In brief, according to the DFT calculation results, MXene plays a critical role in promoting electron/mass transfer at ZnS (111)/MXene heterointerface to achieve excellent lithium storage performance.
Based on the above results, we clearly demonstrate that the 0D-2D ZnS nanodots/MXene hybrid exhibits good lithium storage performance due to the surface dominating lithium storage behavior with excellent electrochemical kinetics. For ZnSMX64, the interfacial bonding of Ti-O-Zn between $\mathrm{ZnS}$ nanodots and MXene nanosheets can immobilize $\mathrm{ZnS}$ nanodots on the surface of MXene stably to prevent detachment of $\mathrm{ZnS}$ nanodots from conducting MXene matrix and boost the interfacial interaction to promote electron/mass transfer. At the ZnS-MXene heterointerface, the 


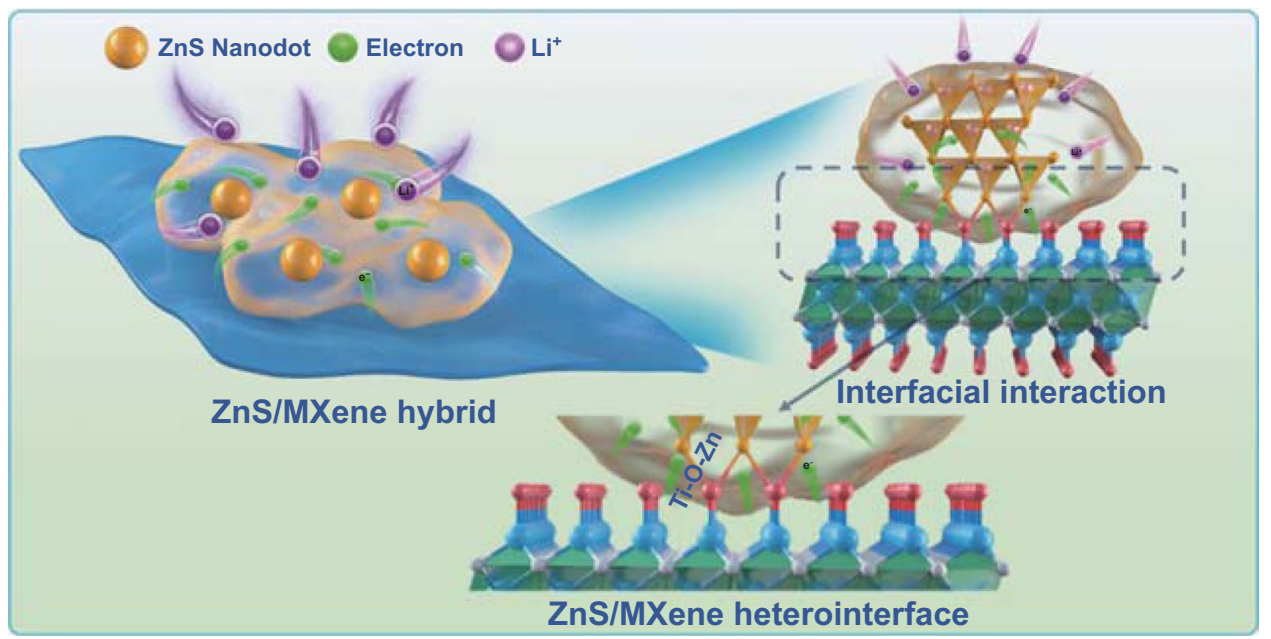

Fig. 8 Schematic illustration for interfacial interaction at ZnS-MXene heterointerface boosting electron transfer and lithium diffusion

strong interfacial interaction will induce electron migration and charge density redistribution from MXene to ZnS. Correspondingly, the ZnS-MXene heterointerface exhibits a high lithium adsorption capability and low diffusion energy barrier. Therefore, the strong interfacial interaction between ZnS nanodots and MXene nanosheets makes ZnSMX64 to exhibit stable cyclability and superior rate performance. The possible interfacial interaction in ZnS-MXene heterointerface for boosting electron transfer and lithium adsorption/ diffusion is schematically illustrated in Fig. 8. In comparison with pure $\mathrm{ZnS}$, the improved lithium storage performance including stable cyclability and high rate performance of ZnSMX64 is ascribed to its unique 0D-2D structure, appropriate $\mathrm{ZnS}$ nanodots loading, and enhanced interfacial electron/mass transfer, which enable effective accommodation of substantial volume variations, stable electron conduction, and fast lithium diffusion.

\section{Conclusions}

In summary, 0D-2D ZnS nanodots/ $\mathrm{Ti}_{3} \mathrm{C}_{2} \mathrm{~T}_{x}$ MXene hybrids were prepared by sulfidation the ZIF-8/MXene precursors to achieve excellent lithium storage performance. Different from the bulk $\mathrm{ZnS}$ with poor lithium storage performance due to substantial volume variation and low lithium storage reversibility, the $\mathrm{ZnS}$ nanodots $/ \mathrm{Ti}_{3} \mathrm{C}_{2} \mathrm{~T}_{x}$ MXene hybrids with the unique 0D-2D structure and strong interfacial interaction can enable immobilization of $\mathrm{ZnS}$ nanodots on MXene substrate, effective accommodation of substantial volume variations, stable electron conduction, and fast lithium diffusion. The as-prepared ZnSMX64 exhibits exceptional lithium storage performance with superior cycle stability $\left(462.8 \mathrm{mAh} \mathrm{g}^{-1}\right.$ after 1000 cycles at $0.5 \mathrm{~A} \mathrm{~g}^{-1}$ without obvious capacity fading) and rate capability (252.5 $\mathrm{mAh} \mathrm{g}^{-1}$ at $2.0 \mathrm{~A} \mathrm{~g}^{-1}$ ), which is ascribed to the surface dominating lithium storage behavior with excellent electrochemical kinetics. DFT calculation result further elucidates the strong lithium adsorption capability, enhanced interfacial electron transfer, and low diffusion energy barrier at the $\mathrm{ZnS}-\mathrm{MX}$ ene heterointerface. Thus, designing a 0D-2D structure by anchoring the nanosized metal sulfide onto MXene nanosheets with strong interfacial interaction is a promising strategy to boost lithium storage performance, which can be extended to other metal dichalcogenides for energy storage application. Our work also deepens the understanding of electrochemical lithium storage mechanisms of $\mathrm{ZnS}$-based anodes and the effect of interfacial interaction on the electron/mass transfer at heterointerface.

Acknowledgements This work is supported by the National Natural Science Foundation of China (21805011, 51902251, 52072021, and U2004212), the State Key Laboratory of Organic-Inorganic Composites (oic-202101010), the Natural Science Foundation of Shaanxi Provincial Department of Education (20JK0753), and the Provincial Joint Fund of Shaanxi (2021JLM-28).

Open Access This article is licensed under a Creative Commons Attribution 4.0 International License, which permits use, sharing, adaptation, distribution and reproduction in any medium or format, 
as long as you give appropriate credit to the original author(s) and the source, provide a link to the Creative Commons licence, and indicate if changes were made. The images or other third party material in this article are included in the article's Creative Commons licence, unless indicated otherwise in a credit line to the material. If material is not included in the article's Creative Commons licence and your intended use is not permitted by statutory regulation or exceeds the permitted use, you will need to obtain permission directly from the copyright holder. To view a copy of this licence, visit http://creativecommons.org/licenses/by/4.0/.

Supplementary Information The online version contains supplementary material available at https://doi.org/10.1007/ s40820-021-00728-x.

\section{References}

1. S. Niu, Z. Wang, M. Yu, M. Yu, L. Xiu et al., MXene-based electrode with enhanced pseudocapacitance and volumetric capacity for power-type and ultra-long life lithium storage. ACS Nano 12, 3928-3937 (2018). https://doi.org/10.1021/ acsnano.8b01459

2. Y. Liu, P. Zhang, N. Sun, B. Anasori, Q. Zhu et al., Selfassembly of transition metal oxide nanostructures on MXene nanosheets for fast and stable lithium storage. Adv. Mater. 30, 1707334 (2018). https://doi.org/10.1002/adma.201707334

3. X. Li, Z. Chen, A. Li, Y. Yu, X. Chen et al., Three-dimensional hierarchical porous structures constructed by two-stage MXene-wrapped Si nanoparticles for Li-Ion batteries. ACS Appl. Mater. Interfaces 12, 48718-48728 (2020). https://doi. org/10.1021/acsami.0c15527

4. D. Wang, C. Zhou, B. Cao, Y. Xu, D. Zhang et al., One-step synthesis of spherical $\mathrm{Si} / \mathrm{C}$ composites with onion-like buffer structure as high-performance anodes for lithium-ion batteries. Energy Storage Mater. 24, 312-318 (2020). https://doi.org/10. 1016/j.ensm.2019.07.045

5. Z. Li, H. Du, J. Lu, L. Wu, L. He et al., Self-assembly of antimony sulfide nanowires on three-dimensional reduced GO with superior electrochemical lithium storage performances. Chem. Phys. Lett. 771, 138529 (2021). https://doi.org/10. 1016/j.cplett.2021.138529

6. X. Wang, S. Zhang, Y. Shan, L. Chen, G. Gao et al., In situ heterogeneous interface construction boosting fast ion/electron transfer for high-performances lithium/potassium storage. Energy Storage Mater. 37, 55-66 (2021). https://doi.org/10. 1016/j.ensm.2021.01.027

7. H. Lu, Y. Zhu, Y. Yuan, L. He, B. Zheng et al., LiFSI as a functional additive of the fluorinated electrolyte for rechargeable Li-S batteries. J. Mater. Sci. Mater. Electron. 32, 58985906 (2021). https://doi.org/10.1007/s10854-021-05310-0

8. S. Liang, S. Zhang, Z. Liu, J. Feng, Z. Jiang et al., Approaching the theoretical sodium storage capacity and ultrahigh rate of layer-expanded $\mathrm{MoS}_{2}$ by interfacial engineering on N-doped graphene. Adv. Energy Mater. 11, 2002600 (2021). https://doi.org/10.1002/aenm.202002600
9. S. Chen, S. Huang, J. Hu, S. Fan, Y. Shang et al., Boosting sodium storage of $\mathrm{Fe}_{1-\mathrm{x}} \mathrm{S} / \mathrm{MoS}_{2}$ composite via heterointerface engineering. Nano-Micro Lett. 11, 80 (2019). https://doi.org/ 10.1007/s40820-019-0311-z

10. T. Zhou, W.K. Pang, C. Zhang, J. Yang, Z. Chen et al., Enhanced sodium-ion battery performance by structural phase transition from two-dimensional hexagonal- $\mathrm{SnS}_{2}$ to orthorhombic-SnS. ACS Nano 8, 8323-8333 (2014). https:// doi.org/10.1021/nn503582c

11. J. Ding, C. Tang, G. Zhu, W. Sun, A. Du et al., Integrating $\mathrm{SnS}_{2}$ quantum dots with nitrogen-doped $\mathrm{Ti}_{3} \mathrm{C}_{2} \mathrm{~T}_{x}$ MXene nanosheets for robust sodium storage performance. ACS Appl. Energy Mater. 4, 846-854 (2021). https://doi.org/10. 1021/acsaem.0c02730

12. L. Yao, Q. Gu, X. Yu, Three-dimensional MOFs@MXene aerogel composite derived MXene threaded hollow carbon confined CoS nanoparticles toward advanced Alkali-Ion batteries. ACS Nano 15, 3228-3240 (2021). https://doi.org/10. 1021/acsnano.0c09898

13. R. Zhang, J. Xu, M. Jia, E. Pan, C. Zhou et al., Ultrafine ZnS quantum dots decorated reduced graphene oxide composites derived from ZIF-8/graphene oxide hybrids as anode for sodium-ion batteries. J. Alloy. Compd. 781, 450-459 (2019). https://doi.org/10.1016/j.jallcom.2018.11.122

14. X. Du, H. Zhao, Z. Zhang, Y. Lu, C. Gao et al., Core-shell structured ZnS-C nanoparticles with enhanced electrochemical properties for high-performance lithium-ion battery anodes. Electrochim. Acta 225, 129-136 (2017). https://doi. org/10.1016/j.electacta.2016.12.118

15. G. Tian, Z. Zhao, A. Sarapulova, C. Das, L. Zhu et al., Understanding the Li-ion storage mechanism in a carbon composited zinc sulfide electrode. J. Mater. Chem. A 7, 15640-15653 (2019). https://doi.org/10.1039/C9TA01382B

16. Y. Zhang, P. Wang, Y. Yin, X. Zhang, L. Fan et al., Heterostructured SnS-ZnS@C hollow nanoboxes embedded in graphene for high performance lithium and sodium ion batteries. Chem. Eng. J. 356, 1042-1051 (2019). https://doi.org/10. 1016/j.cej.2018.09.131

17. Z. Zhang, Y. Huang, X. Liu, C. Chen, Z. Xu et al., Zeolitic imidazolate frameworks derived $\mathrm{ZnS} / \mathrm{Co}_{3} \mathrm{~S}_{4}$ composite nanoparticles doping on polyhedral carbon framework for efficient lithium/sodium storage anode materials. Carbon 157, 244-254 (2020). https://doi.org/10.1016/j.carbon.2019.10.052

18. L. Huang, Y. Zhang, C. Shang, X. Wang, G. Zhou et al., ZnS nanotubes/carbon cloth as a reversible and high-capacity anode material for Lithium-Ion Batteries. ChemElectroChem 6, 461-466 (2019). https://doi.org/10.1002/celc.201801289

19. Y. Feng, Y. Zhang, Y. Wei, X. Song, Y. Fu et al., A ZnS nanocrystal/reduced graphene oxide composite anode with enhanced electrochemical performances for lithium-ion batteries. Phys. Chem. Chem. Phys. 18, 30630-30642 (2016). https://doi.org/10.1039/C6CP06609G

20. H. Liu, H. Du, W. Zhao, X. Qiang, B. Zheng et al., Fast potassium migration in mesoporous carbon with ultrathin framework boosting superior rate performance for high-power 
potassium storage. Energy Storage Mater. 40, 490-498 (2021). https://doi.org/10.1016/j.ensm.2021.05.037

21. B. Cao, H. Liu, B. Xu, Y. Lei, X. Chen et al., Mesoporous soft carbon as an anode material for sodium ion batteries with superior rate and cycling performance. J. Mater. Chem. A 4, 6472-6478 (2016). https://doi.org/10.1039/C6TA00950F

22. W. Zhang, Z. Huang, H. Zhou, S. Li, C. Wang et al., Facile synthesis of $\mathrm{ZnS}$ nanoparticles decorated on defective CNTs with excellent performances for lithium-ion batteries anode material. J. Alloy. Compd. 816, 152633 (2020). https://doi. org/10.1016/j.jallcom.2019.152633

23. Q. Zhu, J. Li, P. Simon, B. Xu, Two-dimensional MXenes for electrochemical capacitor applications: progress, challenges and perspectives. Energy Storage Mater. 35, 630-660 (2021). https://doi.org/10.1016/j.ensm.2020.11.035

24. X. Zang, J. Wang, Y. Qin, T. Wang, C. He et al., Enhancing capacitance performance of $\mathrm{Ti}_{3} \mathrm{C}_{2} \mathrm{~T}_{x}$ MXene as electrode materials of supercapacitor: from controlled preparation to composite structure construction. Nano-Micro Lett. 12, 77 (2020). https://doi.org/10.1007/s40820-020-0415-5

25. P. Zhang, Q. Zhu, Z. Guan, Q. Zhao, N. Sun et al., A flexible Si@C electrode with excellent stability employing an MXene as a multifunctional binder for Lithium-Ion batteries. Chemsuschem 13, 1621-1628 (2020). https://doi.org/ $10.1002 /$ cssc. 201901497

26. N. Sun, Q. Zhu, B. Anasori, P. Zhang, H. Liu et al., MXenebonded flexible hard carbon film as anode for stable $\mathrm{Na} / \mathrm{K}$ Ion storage. Adv. Funct. Mater. 29, 1906282 (2019). https:// doi.org/10.1002/adfm.201906282

27. S. Zhang, H. Liu, B. Cao, Q. Zhu, P. Zhang et al., An MXene/CNTs@P nanohybrid with stable Ti-O-P bonds for enhanced lithium ion storage. J. Mater. Chem. A 7, 2176621773 (2019). https://doi.org/10.1039/C9TA07357D

28. Y. Li, G. Ma, H. Shao, P. Xiao, J. Lu et al., Electrochemical lithium storage performance of molten salt derived $\mathrm{V}_{2} \mathrm{SnC}$ MAX phase. Nano-Micro Lett. 13, 158 (2021). https://doi. org/10.1007/s40820-021-00684-6

29. C. Sun, C. Wu, X. Gu, C. Wang, Q. Wang, Interface engineering via $\mathrm{Ti}_{3} \mathrm{C}_{2} \mathrm{~T}_{x}$ MXene electrolyte additive toward dendrite-free zinc deposition. Nano-Micro Lett. 13, 89 (2021). https://doi.org/10.1007/s40820-021-00612-8

30. J. Yan, C.E. Ren, K. Maleski, C.B. Hatter, B. Anasori et al., Flexible MXene/graphene films for ultrafast supercapacitors with outstanding volumetric capacitance. Adv. Funct. Mater. 27, 1701264 (2017). https://doi.org/10.1002/adfm. 201701264

31. N. Sun, Z. Guan, Q. Zhu, B. Anasori, Y. Gogotsi et al., Enhanced ionic accessibility of flexible MXene electrodes produced by natural sedimentation. Nano-Micro Lett. 12, 89 (2020). https://doi.org/10.1007/s40820-020-00426-0

32. M. Naguib, M. Kurtoglu, V. Presser, J. Lu, J. Niu et al., Twodimensional nanocrystals produced by exfoliation of $\mathrm{Ti}_{3} \mathrm{AlC}_{2}$. Adv. Mater. 23, 4248-4253 (2011). https://doi.org/10.1002/ adma.201102306

33. A. Shayesteh Zeraati, S.A. Mirkhani, P. Sun, M. Naguib, P.V. Braun et al., Improved synthesis of $\mathrm{Ti}_{3} \mathrm{C}_{2} \mathrm{~T}_{x}$ MXenes resulting in exceptional electrical conductivity, high synthesis yield, and enhanced capacitance. Nanoscale 13, 3572-3580 (2021). https://doi.org/10.1039/d0nr06671k

34. Y. Wei, P. Zhang, R.A. Soomro, Q. Zhu, B. Xu, Advances in the synthesis of 2D Mxenes. Adv. Mater. 2103148 (2021). https://doi.org/10.1002/adma.202103148

35. C.J. Zhang, B. Anasori, A. Seral-Ascaso, S. Park, N. McEvoy et al., Transparent, flexible, and conductive 2D titanium carbide (MXene) films with high volumetric capacitance. Adv. Mater. 29, 1702678 (2017). https://doi.org/10.1002/adma. 201702678

36. H. Liu, X. Zhang, Y. Zhu, B. Cao, Q. Zhu et al., Electrostatic self-assembly of 0D-2D $\mathrm{SnO}_{2}$ quantum Dots $/ \mathrm{Ti}_{3} \mathrm{C}_{2} \mathrm{~T}_{x}$ MXene hybrids as anode for lithium-ion batteries. Nano-Micro Lett. 11, 65 (2019). https://doi.org/10.1007/s40820-019-0296-7

37. Y. Zheng, T. Zhou, C. Zhang, J. Mao, H. Liu et al., Boosted charge transfer in $\mathrm{SnS} / \mathrm{SnO}_{2}$ heterostructures: toward high rate capability for sodium-ion batteries. Angew. Chem. Int. Ed. 55, 3408-3413 (2016). https://doi.org/10.1002/anie.201510978

38. M. Zhao, M. Torelli, C.E. Ren, M. Ghidiu, Z. Ling et al., 2D titanium carbide and transition metal oxides hybrid electrodes for Li-ion storage. Nano Energy 30, 603-613 (2016). https:// doi.org/10.1016/j.nanoen.2016.10.062

39. R. Meng, J. Huang, Y. Feng, L. Zu, C. Peng et al., Black phosphorus quantum $\mathrm{Dot} / \mathrm{Ti}_{3} \mathrm{C}_{2} \mathrm{MXene}$ nanosheet composites for efficient electrochemical lithium/sodium-ion storage. Adv. Energy Mater. 8, 1801514 (2018). https://doi.org/10.1002/ aenm.201801514

40. H. Gao, T. Zhou, Y. Zheng, Y. Liu, J. Chen et al., Integrated carbon/red phosphorus/graphene aerogel $3 \mathrm{~d}$ architecture via advanced vapor-redistribution for high-energy sodium-ion batteries. Adv. Energy Mater. 6, 1601037 (2016). https://doi.org/ 10.1002/aenm.201601037

41. Y. Zhang, Z. Mu, J. Lai, Y. Chao, Y. Yang et al., MXene/Si@ $\mathrm{SiO}_{\mathrm{x}} @ \mathrm{C}$ layer-by-layer superstructure with autoadjustable function for superior stable lithium storage. ACS Nano 13, 2167-2175 (2019). https://doi.org/10.1021/acsnano.8b08821

42. X. Hui, R. Zhao, P. Zhang, C. Li, C. Wang et al., Low-temperature reduction strategy synthesized $\mathrm{Si} / \mathrm{Ti}_{3} \mathrm{C}_{2}$ MXene composite anodes for high-performance Li-ion batteries. Adv. Energy Mater. 9, 1901065 (2019). https://doi.org/10.1002/aenm.20190 1065

43. J. Luo, J. Zheng, J. Nai, C. Jin, H. Yuan et al., Atomic sulfur covalently engineered interlayers of $\mathrm{Ti}_{3} \mathrm{C}_{2}$ MXene for ultrafast sodium-ion storage by enhanced pseudocapacitance. Adv. Funct. Mater. 29, 1808107 (2019). https://doi.org/10.1002/ adfm.201808107

44. M. Boota, B. Anasori, C. Voigt, M. Zhao, M.W. Barsoum et al., Pseudocapacitive electrodes produced by oxidant-free polymerization of pyrrole between the layers of $2 \mathrm{D}$ titanium carbide (MXene). Adv. Mater. 28, 1517-1522 (2016). https:// doi.org/10.1002/adma.201504705

45. J. Yoon, I.T. Kim, J. Bae, J. Hur, High-performance ZnS@ graphite composites prepared through scalable high-energy ball milling as novel anodes in lithium-ion batteries. J. Ind. 
Eng. Chem. 76, 258-267 (2019). https://doi.org/10.1016/j.jiec. 2019.03.050

46. T. Hu, J. Wang, H. Zhang, Z. Li, M. Hu et al., Vibrational properties of $\mathrm{Ti}_{3} \mathrm{C}_{2}$ and $\mathrm{Ti}_{3} \mathrm{C}_{2} \mathrm{~T}_{2}(\mathrm{~T}=\mathrm{O}, \mathrm{F}, \mathrm{OH})$ monosheets by first-principles calculations: a comparative study. Phys. Chem. Chem. Phys. 17, 9997-10003 (2015). https://doi.org/ 10.1039/C4CP05666C

47. Y. Xie, Y. Dall Agnese, M. Naguib, Y. Gogotsi, M.W. Barsoum et al., Prediction and characterization of MXene nanosheet anodes for non-lithium-ion batteries. ACS Nano 8, 9606-9615 (2014). https://doi.org/10.1021/nn503921j

48. K.K. Senapati, C. Borgohain, P. Phukan, $\mathrm{CoFe}_{2} \mathrm{O}_{4}-\mathrm{ZnS}$ nanocomposite: a magnetically recyclable photocatalyst. Catal. Sci. Technol. 2, 2361 (2012). https://doi.org/10.1039/c2cy20400b

49. Z. Guan, W. Luo, Z. Zou, Formation mechanism of ZnS impurities and their effect on photoelectrochemical properties on a $\mathrm{Cu}_{2} \mathrm{ZnSnS}_{4}$ photocathode. CrystEngComm 16, 2929 (2014). https://doi.org/10.1039/c3ce42373e

50. B. Cao, H. Liu, P. Zhang, N. Sun, B. Zheng et al., Flexible MXene framework as a fast electron/potassium-ion dual-function conductor boosting stable potassium storage in graphite electrodes. Adv. Funct. Mater. 31, 2102126 (2021). https://doi. org/10.1002/adfm.202102126

51. Q. Zhao, Q. Zhu, J. Miao, P. Zhang, P. Wan et al., Flexible 3D porous MXene foam for high-performance lithium-ion batteries. Small 15, 1904293 (2019). https://doi.org/10.1002/smll. 201904293

52. H. Zhao, Y. Chen, X. Quan, X. Ruan, Preparation of Zndoped $\mathrm{TiO}_{2}$ nanotubes electrode and its application in pentachlorophenol photoelectrocatalytic degradation. Chin. Sci. Bull. 52, 1456-1461 (2007). https://doi.org/10.1007/ s11434-007-0170-8

53. J. Chu, W.A. Wang, J. Feng, C. Lao, K. Xi et al., Deeply nesting Zinc sulfide dendrites in tertiary hierarchical structure for potassium ion batteries: enhanced conductivity from interior to exterior. ACS Nano 13, 6906-6916 (2019). https://doi.org/ 10.1021/acsnano.9b01773

54. D. Fang, S. Chen, X. Wang, Y. Bando, D. Golberg et al., ZnS quantum dots@multilayered carbon: geological-plate-movement-inspired design for high-energy Li-ion batteries. J. Mater. Chem. A 6, 8358-8365 (2018). https://doi.org/10.1039/C8TA01667D

55. H. Wang, K. Xie, Y. You, Q. Hou, K. Zhang et al., Realizing interfacial electronic interaction within $\mathrm{ZnS}$ quantum dots/NrGO heterostructures for efficient $\mathrm{Li}-\mathrm{CO}_{2}$ batteries. Adv. Energy Mater. 9, 1901806 (2019). https://doi.org/10.1002/ aenm.201901806

56. J. Zheng, Y. Wu, Y. Sun, J. Rong, H. Li et al., Advanced anode materials of potassium ion batteries: from zero dimension to three dimensions. Nano-Micro Lett. 13, 12 (2021). https://doi. org/10.1007/s40820-020-00541-y

57. Y. Hwa, J.H. Sung, B. Wang, C. Park, H. Sohn, Nanostructured Zn-based composite anodes for rechargeable Li-ion batteries. J. Mater. Chem. 22, 12767-12773 (2012). https://doi.org/10. 1039/c2jm31776a

58. H. Ding, H.C. Huang, X.K. Zhang, L. Xie, J.Q. Fan et al., Zinc sulfide decorated on nitrogen-doped carbon derived from metal-organic framework composites for highly reversible Lithium-Ion battery anode. ChemElectroChem 6, 5617-5626 (2019). https://doi.org/10.1002/celc.201901568

59. P. Simon, Y. Gogotsi, B. Dunn, Where do batteries end and supercapacitors begin? Science 343, 1210-1211 (2014). https://doi.org/10.1126/science.1249625

60. J. Wang, J. Polleux, J. Lim, B. Dunn, Pseudocapacitive contributions to electrochemical energy storage in $\mathrm{TiO}_{2}$ (Anatase) nanoparticles. J. Phys. Chem. C 111, 14925-14931 (2007). https://doi.org/10.1021/jp074464w

61. B. Cao, Q. Zhang, H. Liu, B. Xu, S. Zhang et al., Graphitic carbon nanocage as a stable and high power anode for potassium-ion batteries. Adv. Energy Mater. 8, 1801149 (2018). https://doi.org/10.1002/aenm.201801149

62. C. Wang, N. Kurra, M. Alhabeb, J. Chang, H.N. Alshareef et al., Titanium carbide (MXene) as a current collector for lithium-ion batteries. ACS Omega 3, 12489-12494 (2018). https://doi.org/10.1021/acsomega.8b02032 